\title{
Stabilizers of direct composition series
}

\author{
Manfred Droste and Rüdiger Göbel
}

\begin{abstract}
Let $R$ be a domain, $V$ a left $R$-module, and $\mathcal{L}$ a composition series of direct summands of $V$. Our main results show that if $U$ is a stabilizer group of $\mathcal{L}$ containing the McLaingroup associated with $\mathcal{L}$, then $U$ determines the chain $(\mathcal{L}, \subseteq)$ uniquely up to isomorphism or anti-isomorphism.
\end{abstract}

\section{Introduction}

In two of his very early papers $[1,2]$ Paul Conrad investigates the group $A$ of $o$-automorphisms of an abelian o-group $G$ with the aim to provide examples of non-abelian o-groups $A$ and to understand how $A$ and $G$ are related. He wants to know: When can $G$ be reconstructed from $A$ ? As a consequence and with the aim to get useful examples (see Theorem 1 in [1] and [2]) Conrad studies groups $A$ of finitary triangular matrices over an infinite dimensional vector space (over the field of rationals $\mathbb{Q}$ ) which now fall under the generic name McLain groups. While McLain groups for obvious reasons were mainly promoted by non-commutative group theorists (see below), we will follow here Conrad's road and investigate the relationship between $A$ and $G$, where $A$ is the automorphism group of the abelian group $G$ controlled by an ordering on $G$ which is expressed as a composition series of $G$. In our case $G$ will be a module over a domain $R$.

Our new results in this paper will also contribute to the following more recent investigations $[4,5,12,13,15,16]$. In the introduction we want to state our main theorem, explain

\footnotetext{
${ }^{0}$ This work is supported by the project No. I-963-98.6/2007 of the German-Israeli Foundation for Scientific Research \& Development.

AMS subject classification: primary: 20B27, 20E15, 20H25 secondary: 06A15

Key words and phrases: automorphism groups of $R$-modules, composition series, McLain-groups, maximal abelian normal subgroups
} 
the techniques of its proof and indicate the connection with these references. As mentioned above, this subject was motivated from group theory by the celebrated result due to McLain establishing the existence of characteristically simple locally finite (thus locally nilpotent) $p$-groups. These groups are subgroups of the group of finitary transformations of an infinite dimensional vector space - in contrast to Paul Conrad - over the field $Z_{p}$ with $p$ elements taking care of a fixed linear ordering of a fixed basis of a vector space of countable dimension. This setting can be seen as a fixed choice of a composition series of this vector space. Thus it is natural to consider an extension of the McLain construction over a more general ordering and an arbitrary (not necessarily commutative) ring $R$. A first investigation, using more general rings can be found in Roseblade [17]. In order to avoid complications it is reasonable to assume that $R$ has no zero-divisors. Then it turns out that generalized McLain groups can be defined in this generality with respect to a fixed composition series $\mathcal{L}$; see Definition 2.1. The fact that over fields we have decomposition of immediate factors of the composition series is reflected in our Definition 2.1 (2) of a direct composition series - by using projectivity of $R$. It follows immediately from the restriction to such composition series, that the $R$-modules $V$ in question (replacing the vector spaces $V$ ) are now submodules of cartesian products $R^{\kappa}$, thus torsion-less, in the sense of Bass. Now McLain groups can be defined as in Definition 3.5. One of the basic question for investigating (generalized) McLain groups is the reconstruction of the composition series $\mathcal{L}$ from the knowledge of the McLain group $M(\mathcal{L})$; it is the analogue of Wedderburn's theorem showing that from the matrix $\operatorname{rings} \operatorname{End}_{K}(V)$ of a finite dimensional vector space the dimension and the ground field $K$ can be recovered. This is also a crucial topic in $[4,5]$ and in Puglisi [15]. We will succeed here in showing the following main theorem. We begin with a few easy remarks and obvious, known definitions. Let $R$ (for the moment) be a domain, i.e. a commutative ring without zero-divisors and $\mathcal{L}=\left\{V_{\lambda} \mid \lambda \in \Lambda\right\}$ a direct composition series of a left $R$-module $V$. We let the index set $\Lambda$ carry the order inherited from the chain $(\mathcal{L}, \subseteq)$. For $\mu \in \Lambda$, let $\mu^{+}$denote the direct successor $\mu$ in $\Lambda$ (if it exists). Then let $\Lambda^{+}=\left\{\lambda \in \Lambda \mid \exists \mu \in \Lambda: \lambda=\mu^{+}\right\}$. We say that $h \in \operatorname{End}_{R} V$ stabilizes $\mathcal{L}$ if $V_{\mu^{+}} h \subseteq V_{\mu}$ for each $\mu \in \Lambda$. Let

$$
\mathcal{G}(\mathcal{L})=\left\{g=1+a \in \operatorname{Aut}_{R}(V) \mid \text { a stabilizes } \mathcal{L}\right\},
$$

the stabilizer group of $\mathcal{L}$. It follows from the definitions that the generalized McLain group is a subgroup of the stabilizer group.

Theorem 1.1. Let $R$ is any domain. Let $\mathcal{L}_{1}, \mathcal{L}_{2}$ be two direct composition series. Let 
$M\left(\mathcal{L}_{i}\right) \subseteq U_{i} \subseteq \mathcal{G}\left(\mathcal{L}_{i}\right)$ for $i=1,2$, and assume that $U_{1} \cong U_{2}$. Then the chains $\left(\mathcal{L}_{1}, \subseteq\right)$ and $\left(\mathcal{L}_{2}, \subseteq\right)$ are either isomorphic or anti-isomorphic.

This result comes in three parts. We must distinguish the cases when $\mathcal{L}^{*}$, the direct composition series without $0, V$, the smallest and the largest element, has no smallest or no largest element, when $\mathcal{L}^{*}$ is bounded and $\operatorname{char}(R) \neq 2$, and when $\mathcal{L}^{*}$ is bounded and $\operatorname{char}(R)=2$, see Theorems 4.11, 6.5 and 6.9. The proof is based on the fact that the maximal normal abelian subgroups of $U_{1}$ must be mapped bijectively onto the corresponding subgroups of $U_{2}$. This leads to the order-theoretic normal subgroups of $U_{1}$ which allow us to recover the betweenness relation on $\mathcal{L}_{1}$ when $\mathcal{L}_{1}^{*}$ is not bounded. In case $\mathcal{L}_{1}^{*}$ is bounded and $\operatorname{char}(R) \neq 2$, we need to employ the maximal intersection groups (groups maximal among the intersections of pairs of distinct maximal abelian normal subgroups). We also consider those maximal abelian normal subgroups which do not contain a maximal intersection group. The case of characteristic two with bounded direct composition series $\mathcal{L}_{1}^{*}$ requires even further algebraic information transported from $U_{1}$ to $U_{2}$ by the group isomorphism. For this case, we also consider maximal intersections of pairs of distinct maximal intersection groups. Investigation of these classes of abelian normal subgroups leads to the above theorem. We do not know if the case of anti-isomorphisms between $\mathcal{L}_{1}$ and $\mathcal{L}_{2}$ can occur. In the particular situation of fields which are not of characteristic two, Puglisi [15] is able to exclude this case using heavily dimension arguments and deep group theoretic results. Thus it seems very likely that anti-isomorphisms cannot come up in general. Also for McLain groups defined directly on a linear ordering as in [4] it can be shown that an isomorphism between those McLain groups induces an order-isomorphism or anti-isomorphism of the ordering.

\section{Basic Constructions}

Let $R$ be any (not necessarily commutative) ring with $0 \neq 1$ and without zero-divisors. Moreover, let $V$ be a left $R$-module and $\operatorname{Aut}_{R} V$ its group of $R$-automorphisms with $1 \in$ $\operatorname{Aut}_{R} V$ the identity on $V$. Then we consider

$$
\operatorname{FGL}(V, R)=\left\{g \in \operatorname{Aut}_{R} V \mid \operatorname{rk}(V(g-1))<\infty\right\} .
$$

This is a normal subgroup of $\operatorname{Aut}_{R} V$ and obviously

$$
\operatorname{FGL}(V, R)=(1+\operatorname{Fin} V) \cap \operatorname{Aut}_{R} V,
$$


where Fin $V=\left\{\sigma \in \operatorname{End}_{R} V \mid \operatorname{rk} V \sigma<\infty\right\}$ is a useful ideal of the endomorphism ring $\operatorname{End}_{R} V$ in connection with realization theorems of algebras, see [9]. If $R$ is commutative, then $\operatorname{End}_{R} V$ is an $R$-algebra and Fin $V$ is a two sided ideal of this algebra. An element $g \in \operatorname{Aut}_{R} V$ is called unipotent if there is an $n \in \mathbb{N}$ such that $(g-1)^{n}=0$ in $\operatorname{End}_{R} V$. If $H \subseteq \operatorname{Aut}_{R} V$ consists of unipotent elements only, then $H$ is said to be unipotent. It is natural to relate unipotent subgroups of Aut $V$ to stabilizers of composition series of $V$.

Definition 2.1. Let $R$ be a ring without zero-divisors and $V$ a left $R$-module. A family $\mathcal{L}=\left\{V_{\lambda} \mid \lambda \in \Lambda\right\}$ of submodules of $V$ is a composition series if the following conditions are satisfied.

(1) $\mathcal{L}$ is linearly ordered under inclusion and contains 0 and $V$.

(2) $\mathcal{L}$ is closed under arbitrary unions and intersections.

(3) If $V_{\lambda}$ is a direct successor of $V_{\mu}$ in $\mathcal{L}$ then $V_{\lambda} / V_{\mu} \cong R$.

(4) $\mathcal{L}$ is maximal with respect to (1), (2), and (3).

Note that any composition series is closed under unions and intersections. Also observe that if $\lambda, \mu \in \Lambda$ and $V_{\lambda}$ is a direct successor of $V_{\mu}$, then $V_{\mu} \sqsubset V_{\lambda}$ (a direct summand), since $R$ is projective. This implies that if $\mathcal{L}$ is an ascending (i.e., well-ordered) composition series, then $\mathcal{L}$ is a direct composition series. Hence initial segments of $\mathcal{L}$ are also composition series.

We call $\mathcal{L}$ a direct composition series if the elements of $\mathcal{L}$ are direct summands of $V$. Next we show that for composition series over domains the converse of (3) holds.

Proposition 2.2. Let $R$ be a domain, $V$ a left $R$-module and $\mathcal{L}$ a composition series. If $V_{\mu} \subset V_{\lambda}$ in $\mathcal{L}$ with $V_{\lambda} / V_{\mu} \cong R$, then $V_{\lambda}$ is a direct successor of $V_{\mu}$ in $\mathcal{L}$.

Proof. Let $V_{\mu} \subseteq W \subset V_{\lambda}$ in $\mathcal{L}$ with $V_{\lambda} / V_{\mu} \cong R$. Since $\mathcal{L}$ is a composition series, we can find $W \subseteq U^{\prime} \subset U \subseteq V_{\lambda}$ in $\mathcal{L}$ such that $U$ is a direct successor of $U^{\prime}$ in $\mathcal{L}$. Thus $U / U^{\prime} \cong R$ and so $U=U^{\prime} \oplus R$. Consider $\left(U^{\prime} / V_{\mu}\right) \oplus R \cong\left(U^{\prime} \oplus R\right) / V_{\mu} \subseteq V_{\lambda} / V_{\mu} \cong R$, which represents a direct sum of ideals of the commutative ring $R$. But $R$ has no zero-divisors. It follows that $U^{\prime}=V_{\mu}$. Hence $W=V_{\mu}$.

We let $\Lambda$ carry the natural induced ordering defined by $\mu \leq \lambda$ iff $V_{\mu} \subseteq V_{\lambda}$ for $\lambda, \mu \in \Lambda$. We write $\lambda=\mu^{+}$(or $\mu \succ \lambda$ ) if $\lambda$ is the direct successor of $\mu$ in $\Lambda$, that is, $\mu<\lambda$ and there is 
no $\rho \in \Lambda$ with $\mu<\rho<\lambda$. Put

$$
\Lambda^{+}=\left\{\lambda \in \Lambda \mid \exists \mu \in \Lambda: \lambda=\mu^{+}\right\} .
$$

Moreover, let

$$
V_{\lambda}^{*}=V_{\lambda} \backslash V_{\lambda}^{-} \text {for } \lambda \in \Lambda^{+} \text {, and } V_{\lambda}^{-}=\bigcup_{\mu<\lambda} V_{\mu} \text { for } \lambda \in \Lambda .
$$

Note that $V_{\lambda}=V_{\lambda}^{-}$if $\lambda=\sup \{\mu \mid \mu<\lambda\}$, and if $\lambda \in \Lambda^{+}$, then $V_{\lambda} / V_{\lambda}^{-} \cong R$. We will often use that whenever $0 \neq v \in V$, then $v \in V_{\lambda}^{*}$ for some $\lambda \in \Lambda^{+}$. A left $R$-module $V$ is called torsionless if $V$ embeds into some product $R^{\kappa}$.

Proposition 2.3. Let $R$ be a ring without zero-divisors and $V$ a left $R$-module.

(a) If $V$ has a direct composition series, then $V$ is torsionless.

(b) If $R$ is principal ideal domain and $V$ is torsionless, then $V$ has a direct composition series. Moreover this series can be chosen to be descending, i.e. anti-isomorphic to an ordinal.

Proof. (a) Let $\mathcal{L}=\left\{V_{\lambda} \mid \lambda \in \Lambda\right\}$ be a direct composition series of $V$. For each $\lambda \in \Lambda^{+}$ choose a decomposition $V=V_{\lambda} \oplus C_{\lambda}$ and let $\pi_{\lambda}: V \rightarrow V_{\lambda}$ be the projection modulo $C_{\lambda}$ and $\rho_{\lambda}: V_{\lambda} \rightarrow V_{\lambda} / V_{\lambda}^{-}$be the canonical projection. Put $\sigma_{\lambda}=\pi_{\lambda} \rho_{\lambda}$. Then

$$
\sigma=\prod_{\lambda \in \Lambda^{+}} \sigma_{\lambda}: V \rightarrow \prod_{\lambda \in \Lambda^{+}} V_{\lambda} / V_{\lambda}^{-}
$$

is an embedding, because if $0 \neq v \in V$, then $v \in V_{\lambda}^{*}$ for some $\lambda \in \Lambda^{+}$, hence $v \notin \operatorname{ker} \sigma_{\lambda}$, thus ker $\sigma=0$. Since $\prod_{\lambda \in \Lambda^{+}} V_{\lambda} / V_{\lambda}^{-} \cong R^{\Lambda^{+}}$, the claim follows.

(b) Since $V$ is torsionless, we can assume that $V \subseteq R^{\kappa}$ for some cardinal $\kappa$. Write $R^{\kappa}=$ $\prod_{i \in \kappa} e_{i} R$ and put $N_{\lambda}=\prod_{\lambda \leq i \in \kappa} e_{i} R$ and $V_{\lambda}=V \cap N_{\lambda}(\lambda \in \kappa)$. It follows that $\mathcal{L}=\left\{V_{\lambda} \mid \lambda \in \kappa\right\}$ is descending and each $V_{\lambda}$ is a direct summand of $V$. We show that $\mathcal{L}$ satisfies condition (2). Assume $\lambda, \mu \in \kappa$ such that $V_{\lambda}$ is a direct successor of $V_{\mu}$ in $\mathcal{L}$. Choose a minimal $\lambda^{\prime} \in \kappa$ such that $V_{\lambda}=V_{\lambda^{\prime}}$. By definition of the $V_{\nu}(\nu \in \kappa), \mathcal{L}$ is closed under intersections. Thus there is a maximal $\mu^{\prime} \in \kappa$ with $V_{\mu}=V_{\mu^{\prime}}$. Then $\mu^{\prime}$ is a direct successor of $\lambda^{\prime}$ in $\kappa$. Hence

$$
0 \neq V_{\lambda} / V_{\mu}=V_{\lambda^{\prime}} / V_{\mu^{\prime}}=\left(V \cap N_{\lambda^{\prime}}\right) /\left(V \cap N_{\mu^{\prime}}\right) \cong N_{\lambda^{\prime}} / N_{\mu^{\prime}} \cong R
$$


Since $R$ is a principal ideal domain, we obtain $V_{\lambda} / V_{\mu} \cong R$. Hence (1) and (2) hold.

Now choose a composition series $\mathcal{L}^{\prime}$ such that $\mathcal{L} \subseteq \mathcal{L}^{\prime}$. Consider any $\lambda, \mu \in \kappa$ such that $V_{\lambda}$ is a direct successor of $V_{\mu}$ in $\mathcal{L}$. Then $V_{\lambda} / V_{\mu} \cong R$ by (2). Hence, by Proposition $2.3, V_{\lambda}$ is a direct successor of $V_{\mu}$ in $\mathcal{L}^{\prime}$. Since $\mathcal{L}$ is descending, it follows that $\mathcal{L}=\mathcal{L}^{\prime}$.

In view of Proposition 2.3(b), we note that $V$ in general does not have an ascending (i.e. well-ordered) composition series as the following result shows.

Theorem 2.4. Let $\kappa$ be an infinite cardinal and $R$ a countable principal ideal domain. Then the following are equivalent:

(1) $R$ is a field.

(2) $R^{\kappa}$ has an ascending direct composition series.

Proof. (1) $\longrightarrow(2)$ : If $R$ is a field, then $R^{\kappa}$ is a vector space over $R$ of dimension $2^{\kappa}$. A well-ordering of a basis induces an ascending direct composition series.

$(2) \longrightarrow(1)$ : If $R$ is not a field, then $R$ is slender, see Eklof and Mekler [6, p. 64, Corollary 2.4] or Göbel and Trlifaj [9]. Suppose $V=R^{\kappa}$ has an ascending direct composition series $V_{\lambda}(\lambda \in \Lambda)$, where $\Lambda$ is an ordinal. Since $|R|<|V|=2^{\kappa}$ and $\left|V_{\lambda}\right| \leq \aleph_{0}$ for all $\lambda<\omega_{1}$ we have $\omega_{1} \in \Lambda$ and $\left|V_{\omega_{1}}\right|=\aleph_{1}$. Also $V_{\omega_{1}} \sqsubset R^{\kappa}$, so by Nunke [14, p. 69, Theorem 5a] and a slight extension (replacing $\mathbb{Z}$ by $R$ ) we obtain $V_{\omega_{1}} \cong R^{\rho}$ for some cardinal $\rho$. If follows that $\aleph_{1}=2^{\rho}$, hence $\rho=\omega$ and CH holds. Express $V_{\omega_{1}}=\prod_{i \in \omega} R e_{i}$. Also, $\left\{V_{\lambda} \mid \lambda \in \omega_{1}\right\}$ is a composition series of $V_{\omega_{1}}$. Since $\operatorname{cf}\left(\omega_{1}\right)=\omega_{1}$ we can find $\lambda \in \omega_{1}$ such that $\left\{e_{i} \mid i \in \omega\right\} \subseteq V_{\lambda}$. Write $V_{\omega_{1}}=V_{\lambda} \oplus C_{\lambda}$ and let $\pi: V_{\omega_{1}} \rightarrow C_{\lambda}$ be the canonical projection. From $e_{i} \pi=0$ for all $i \in \omega$ it follows that $\pi$ induces $\bar{\pi}:\left(\prod_{i \in \omega} R e_{i}\right) /\left(\bigoplus_{i \in \omega} R e_{i}\right) \rightarrow C_{\lambda}$ with $\operatorname{Im}(\pi)=\operatorname{Im}(\bar{\pi})$. However $\prod_{i \in \omega} R e_{i} / \bigoplus_{i \in \omega} R_{e_{i}}$ is cotorsion by Hulanicki, see Fuchs [7, vol.1, p. 176, Corollary 42.2]. On the other hand $C_{\lambda} \subset R^{\omega}$ and therefore cotorsion-free, see Eklof and Mekler [6, p.138, Theorem 2.9]. Hence

$$
0=\operatorname{Im}(\bar{\pi})=\operatorname{Im}(\pi)=C_{\lambda} \text { and } V_{\omega_{1}}=V_{\lambda} .
$$

But $\lambda \in \omega_{1}$ and $V_{\lambda}$ is countable, a contradiction.

Next we consider endomorphisms stabilizing $\mathcal{L}$. 
Definition 2.5. Let $R$ be a ring without zero-divisors and $\mathcal{L}=\left\{V_{\lambda} \mid \lambda \in \Lambda\right\}$ a direct composition series of a left $R$-module $V$.

(a) If $g \in \operatorname{Aut}_{R}(V)$, then $a=g-1 \in \operatorname{End}_{R} V$; we often write $g=1+a$.

(b) We say that $h \in \operatorname{End}_{R} V$ stabilizes $\mathcal{L}$ if $V_{\lambda} h \subseteq V_{\lambda}^{-}$for each $\lambda \in \Lambda^{+}$.

(c) Let $\mathcal{G}(\mathcal{L})=\left\{g=1+a \in \operatorname{Aut}_{R}(V) \mid \forall \lambda \in \Lambda^{+}: V_{\lambda} a \subseteq V_{\lambda}^{-}\right\}$, the stabilizer group of $\mathcal{L}$.

Proposition 2.6. $\mathcal{G}(\mathcal{L})$ is a group.

Proof. Let $g=1+a \in \mathcal{G}(\mathcal{L})$. We first show that $V_{\lambda} g=V_{\lambda}$ for each $\lambda \in \Lambda$. The inclusion $V_{\lambda} g \subseteq V_{\lambda}$ is clear. Now let $v \in V_{\lambda}^{*}$ for some $\lambda \in \Lambda^{+}$. Since $g \in \operatorname{Aut}_{R} V$, there is $u \in V$ with $v=u g=u+u a$. Then $u \in V_{\mu}^{*}$ for some $\mu \in \Lambda^{+}$, and $u a \in V_{\mu}^{-}$. So $v \in V_{\mu}^{*}$, showing $\mu=\lambda$ and $v=u g \in V_{\lambda} g$.

Now $g^{-1}=1+b$ with $b=g^{-1}-1$, and by the above, $V_{\lambda} b \subseteq V_{\lambda}$ for each $\lambda$. We claim that $V_{\lambda} b \subseteq V_{\lambda}^{-}$for each $\lambda \in \Lambda^{+}$. Since $1=g g^{-1}=(1+a)(1+b)=1+a+b+a b$, we have $0=a+b+a b$. Now if $v \in V_{\lambda}$, then $v b=-v a-v a b$. We get $v a \in V_{\lambda}^{-}$by assumption, so $v a b \in V_{\lambda}^{-}$by the above, thus $v b \in V_{\lambda}^{-}$as needed.

We will write $\operatorname{FG}(\mathcal{L})=\mathcal{G}(\mathcal{L}) \cap \operatorname{FGL}(V, R)$ for the finitary stabilizer of $\mathcal{L}$.

Example 2.7. We give an example of a maximal series $\mathcal{L}$ of submodules of $V$ with $\mathrm{FG}(\mathcal{L})$ not unipotent. Choose $J_{p}=R=V$, the ring of p-adic integers, and $V_{n}=p^{n} J_{p}(n \in \omega)$. Then $\mathcal{L}=\left\{V_{n} \mid n \in \omega\right\}$ is a maximal descending series of $J_{p}$-submodules with $V_{n} / V_{n+1} \cong \mathbb{Z}_{p}$, and $\mathrm{FG}(\mathcal{L})=1+p J_{p}$.

Proof. Note that $J_{p}=$ End $J_{p}$ by scalar multiplication. If $g=1+p a \in 1+p J_{p}$ then $g$ is invertible because $p J_{p}$ is the Jacobson radical of $J_{p}$. Hence $g$ represents an element in $\operatorname{Aut}_{R} V$. Moreover $V_{n} p a \subseteq V_{n+1}$ for all $n \in \omega$ and all $J_{p^{-}}$submodules have rank 1 , thus $g=1+p a \in \mathrm{FG}(\mathcal{L})$.

Conversely, let $g=1+a \in \mathrm{FG}(\mathcal{L})$. Then $J_{p} a \subseteq p J_{p}$ and $a \in p J_{p}$ is immediate.

Note that $J_{p}$ is a domain, hence $1+p J_{p}$ has no non-trivial unipotent elements. 


\section{Relating $\mathcal{G}(\mathcal{L})$ and McLain-groups}

In all of this section let $R$ be a ring without zero-divisors and $\mathcal{L}=\left\{V_{\lambda} \mid \lambda \in \Lambda\right\}$ a direct composition series of a left $R$-module $V$. Here we will investigate the relationship between the stabilizer group $\mathcal{G}(\mathcal{L})$ and related McLain-groups.

Given $g=1+a \in \operatorname{Aut}_{R} V$, we put

$$
[g]=[a]=\left\{(\alpha, \beta) \in \Lambda^{+} \times \Lambda^{+} \mid \exists v \in V_{\alpha}^{*}: v a \in V_{\beta}^{*}\right\}
$$

the support of $g$ respectively $a$. We also put

$$
\begin{aligned}
& {[g]_{1}=[a]_{1}=\left\{\alpha \in \Lambda^{+} \mid \exists \beta \in \Lambda^{+}:(\alpha, \beta) \in[g]\right\},} \\
& {[g]_{2}=[a]_{2}=\left\{\beta \in \Lambda^{+} \mid \exists \alpha \in \Lambda^{+}:(\alpha, \beta) \in[g]\right\},}
\end{aligned}
$$

the 1-support resp. 2-support of $g$ resp. a.

We often write $g^{-1}=1+a^{*}$. Then $a+a^{*}+a a^{*}=a+a^{*}+a^{*} a=0$. Subsequently, the symbols $\alpha, \beta, \gamma, \lambda, \mu, \nu, \omega$ will always denote elements from $\Lambda^{+}$.

Lemma 3.1. Let $g=1+a \in \mathcal{G}(\mathcal{L}),(\alpha, \beta) \in[a]$ and $\alpha^{\prime}>\alpha$. Then $\left(\alpha^{\prime}, \beta^{\prime}\right) \in[a]$ for some $\beta^{\prime} \geq \beta$.

Proof. Choose any $u^{\prime} \in V_{\alpha^{\prime}}^{*}$. If $u^{\prime} a \in V_{\beta}^{*}$, we are done. Now let $u^{\prime} a=0$ or $u^{\prime} a \in V_{\gamma}^{*}$ for some $\gamma \neq \beta$. Choose $u \in V_{\alpha}^{*}$ with $u a \in V_{\beta}^{*}$. Then $u^{\prime}+u \in V_{\alpha^{\prime}}^{*}$ and $\left(u^{\prime}+u\right) a=u^{\prime} a+u a \in$ $V_{\gamma}^{*} \cup V_{\beta}^{*}$, showing $\left(\alpha^{\prime}, \gamma\right) \in[a]$ if $\gamma>\beta$, and $\left(\alpha^{\prime}, \beta\right) \in[a]$ otherwise.

Lemma 3.2. Let $g=1+a \in \mathcal{G}(\mathcal{L})$ and $g^{-1}=1+a^{*}$. Then $[a]=\left[a^{*}\right]$. Moreover, if $\beta \succ \gamma$ and $v \in V_{\beta}$, then $v a \equiv-v a^{*} \bmod V_{\gamma}^{-}$.

Proof. We have $a+a^{*}+a a^{*}=0$. Let $(\alpha, \beta) \in[a]$. Choose $u \in V_{\alpha}^{*}$ with $u a \in V_{\beta}^{*}$. Then $u a^{*}=-u a-u a a^{*}$. Since $(u a) a^{*} \in V_{\beta}^{-}$, we have $u a^{*} \in V_{\beta}^{*}$ and so $(\alpha, \beta) \in\left[a^{*}\right]$. Now let $\beta \succ \gamma$ and $v \in V_{\beta}$. Then $V_{\beta} a a^{*} \subseteq V_{\gamma}^{-}$. Hence $v a+v a^{*} \equiv 0 \bmod V_{\gamma}^{-}$.

Lemma 3.3. Let $g=1+a \in \operatorname{Aut}_{R}(V)$. Then $g \in \mathcal{G}(\mathcal{L})$ iff for all $(\alpha, \beta) \in[g]$ we have $\alpha>\beta$. 
Proof. The 'only-if-part' is obvious. For the converse, let $0 \neq v \in V$ with $v a \neq 0$. Then there are $\alpha, \beta \in \Lambda^{+}$such that $v \in V_{\alpha}^{*}$ and $v a \in V_{\beta}^{*}$. Thus $(\alpha, \beta) \in[a]$, showing $\alpha>\beta$ and $v a \in V_{\alpha}^{-}$.

Lemma 3.4. Given a homomorphism $h: V \rightarrow V$ with $V_{\lambda} h \subseteq V_{\lambda}^{-}$for all $\lambda \in \Lambda^{+}$. Let $g=1+h$. Then:

(a) $g$ is a monomorphism.

(b) Assume $\forall v \in V \exists n \in \mathbb{N}: v h^{n+1}=0$. Then $g \in \operatorname{Aut}_{R}(V)$ and

$$
g^{-1}=1-h+h^{2}-\cdots \pm h^{n} \mp \ldots
$$

(c) If $\Lambda$ is well-ordered, then $g \in \operatorname{Aut}_{R}(V)$.

Proof. (a) We have $u g=u+u h$ and $v g=v+v h$. If $u g=v g$, then $u-v=(v-u) h$. If $u \neq v$, there is $\alpha \in \Lambda^{+}$with $u-v \in V_{\alpha}^{*}$ and so $(u-v) h \in V_{\alpha}^{-}$, a contradiction.

(b) Immediate by $(1+h) g^{-1}=1$.

(c) By assumption on $h$ and $\Lambda$, there is no $v \in V$ with $v h^{n} \neq 0$ for each $n \in \mathbb{N}$. Now apply (b).

Next we define particular group elements stabilizing $\mathcal{L}$.

Definition 3.5. Let $\alpha, \beta \in \Lambda^{+}$with $\alpha>\beta$. Choose any elements $u \in V_{\alpha}^{*}$ and $v \in V_{\beta}^{*}$ with $V_{\alpha}=R u \oplus V_{\alpha}^{-}$. Write $V=\left(R u \oplus V_{\alpha}^{-}\right) \oplus C$. Define $h_{\alpha \beta}: V \rightarrow V$ by $u h_{\alpha \beta}=v$ and $\left(V_{\alpha}^{-} \oplus C\right) h_{\alpha \beta}=0$. Then $h_{\alpha \beta}^{2}=0$, so $g_{\alpha \beta}=1+h_{\alpha \beta} \in \operatorname{Aut}_{R}(V)$ by Lemma 3.4, so $g_{\alpha \beta} \in \mathcal{G}(\mathcal{L})$ by choice of $h_{\alpha \beta}$, and $g_{\alpha \beta}^{-1}=1-h_{\alpha \beta}$.

All elements $g_{\alpha \beta}=1+h_{\alpha \beta}$ arising this way (i.e., by suitably chosen $u, v, C$ ) will be called McLain-elements of type $(\alpha, \beta)$. We put

$$
\left.M(\mathcal{L})=\left\langle g_{\alpha \beta}\right| g_{\alpha \beta} \text { a McLain-element of type }(\alpha, \beta), \alpha>\beta \text { in } \Lambda^{+}\right\rangle,
$$

the McLain-group of $\mathcal{L}$. Thus $M(\mathcal{L}) \subseteq \mathcal{G}(\mathcal{L})$.

Lemma 3.6. Let $\alpha>\beta$. Then $\left[h_{\alpha \beta}\right]=\left\{\left(\alpha^{\prime}, \beta\right) \mid \alpha^{\prime} \geq \alpha\right\}$. However, for each $\alpha^{\prime}>\alpha$, there exists $u^{\prime} \in V_{\alpha^{\prime}}^{*}$ with $u^{\prime} h_{\alpha \beta}=0$. We have $V h_{\alpha \beta}=R v \subseteq V_{\beta}$. 
Proof. Let $h_{\alpha \beta}$ arise from the decomposition $V=\left(R u \oplus V_{\alpha}^{-}\right) \oplus C$ with $u \in V_{\alpha}^{*}$ and $v \in V_{\beta}^{*}$. Let $\left(\alpha^{\prime}, \beta\right) \in\left[h_{\alpha \beta}\right]$. Choose any $w \in V_{\alpha^{\prime}}^{*}$ with $w h_{\alpha \beta} \neq 0$. Then $w \notin V_{\alpha}^{-}$, so $\alpha^{\prime} \geq \alpha$ and $w=x u+w^{\prime}$ for some $x \in R \backslash\{0\}$ and $w^{\prime} \in V_{\alpha}^{-} \oplus C$. Thus $w h_{\alpha \beta}=x v \in V_{\beta}^{*}$ by $x \neq 0$. Hence $\left[h_{\alpha \beta}\right] \subseteq\left\{\left(\alpha^{\prime}, \beta\right) ; \alpha^{\prime} \geq \alpha\right\}$. Conversely, let $\alpha^{\prime}>\alpha$. By Lemma 3.1, we have $\left(\alpha^{\prime}, \beta^{\prime}\right) \in\left[h_{\alpha \beta}\right]$ for some $\beta^{\prime}$. By what we have already shown, then $\beta^{\prime}=\beta$. Moreover let $u^{\prime}=w^{\prime}=w-x u$. Then $u^{\prime} \in V_{\alpha^{\prime}}^{*}$ and $u^{\prime} \in V_{\alpha}^{-} \oplus C$, so $u^{\prime} h_{\alpha \beta}=0$. The final statement is clear.

The following example, an immediate consequence of the main result in Göbel, Wald [10, Theorem, p. 271], illustrates the assumptions of the next Proposition 3.7. There is an abelian group $V$ of cardinality $2^{\aleph_{0}}$ with the following properties.

(i) $\bigoplus_{n \in \omega} \mathbb{Z} e_{n} \subseteq V \subseteq \prod_{n \in \omega} \mathbb{Z} e_{n}$

(ii) End $V=\mathbb{Z} \oplus$ Fin $V$ with Fin $V=\{\varphi \in$ End $V \mid \operatorname{rk} \varphi<\infty\}$

(iii) $V$ is slender.

(A similar result, but replacing $\omega$ in (i) by arbitrary uncountable, regular cardinals follows from [3], see also [9].)

Since $P=\prod_{n \in \omega} \mathbb{Z} e_{n}$ is $\aleph_{1}$-free it is clear that the rank-condition in (ii) can be replaced by the requirement that $\operatorname{Im} \varphi$ is finitely generated (and free). It also follows that Fin $V$ is the collection of endomorphisms $\varphi$ that extend (uniquely) to $\varphi: P \longrightarrow V$ with $e_{i} \varphi=0$ for almost all $i \in \omega$. (This is related to condition (iii).) Thus any element of Fin $V$ can be expressed as a finite sum of endomorphisms $\psi_{n}$ with $e_{i} \psi_{n}=0$ (for $i \neq n$ ) and acting non-trivially only on $\mathbb{Z} e_{n}$. The units of End $V$ are the automorphisms of $V$, i.e. Aut $V=\left\{ \pm \operatorname{id}_{V}+f \mid f \in \operatorname{Fin} V\right\}$.

If $P_{n}=\prod_{i \geq n} \mathbb{Z} e_{i}$, then $V_{n}=V \cap P_{n}(n<\omega)$ represents a descending composition series $\mathcal{L}=\left\{V_{n} \mid n<\omega\right\}$ of $V$ of order type $\omega^{*}$ with $\bigcap_{n \in \omega} V_{n}=0$ such that $V_{n}=\mathbb{Z} e_{n} \oplus V_{n+1}$. The family $F=\left\{\varphi \in\right.$ End $\left.V \mid V_{n} \varphi \subseteq V_{n+1}\right\}$ of all endomorphisms of $V$ which stabilize $\mathcal{L}$ is a subring (without a 1 ) of the two-sided ideal Fin $V$ of End $V=\mathbb{Z} \oplus$ Fin $V$. Thus $F$ is generated (as a ring) by all $\varphi \in$ Fin $V$ shifting elements non-trivially only on $\mathbb{Z} e_{n}$ for some $n \in \omega$, this means $e_{i} \varphi=0$ if $i \neq n$ and $e_{n} \varphi \in V_{n+1}$. In particular $\mathcal{G}(\mathcal{L})=\left\{ \pm \operatorname{id}_{V}+a \mid a \in F\right\}$. Applying Definition 3.5, and the remarks above it follows by simple arguments from linear algebra (similar to the proof of the finite case in Proposition 3.7) that $M(\mathcal{L})=\mathcal{G}(\mathcal{L})$. 
This example shows that we cannot expect that the next proposition (in case of descending chains) can hold for domains $R$ (even if $R=\mathbb{Z}$ ). To characterize finite composition series we must restrict to fields $R$ or must avoid descending chains as above.

Proposition 3.7. If $R$ is a domain and $\mathcal{L}$ is ascending with $M(\mathcal{L})=\mathcal{G}(\mathcal{L})$, then $\mathcal{L}$ is finite, and conversely, if $\mathcal{L}$ is finite, then $M(\mathcal{L})=\mathcal{G}(\mathcal{L})$. In particular, if $R$ is a field, then we have $M(\mathcal{L})=\mathcal{G}(\mathcal{L})$ if and only if $\mathcal{L}$ is finite.

Proof. First let $R$ be a domain, and assume that $\mathcal{L}$ is finite. We may also assume that $V_{j}=\bigoplus_{i=1}^{j} R e_{i}$ for $1 \leq j \leq n, V=V_{n}$ and $V_{0}=\{0\}$. Choose any $1 \neq g=1+a \in \mathcal{G}(\mathcal{L})$. We claim that $g \in M(\mathcal{L})$.

Choose $i \leq n$ minimal with $e_{i} a \neq 0$, say $e_{i} a \in V_{j}^{*}$ with $1 \leq j<i$. Define $h_{i j}: V \longrightarrow V$ by $e_{i} h_{i j}=-e_{i} a$ and $\left(\bigoplus_{k \neq i} V_{k}\right) h_{i j}=0$. Then $1+h_{i j} \in M(\mathcal{L})$ and

$$
(1+a)\left(1+h_{i j}\right)=1+a+h_{i j}+a h_{i j}=1+a^{\prime}=g^{\prime} \in \mathcal{G}(\mathcal{L})
$$

satisfies $e_{k} a^{\prime}=0$ for each $1 \leq k \leq i$. By induction, we have $g^{\prime} \in M(\mathcal{L})$ and hence $g \in M(\mathcal{L})$.

Now assume that $\mathcal{L}$ is ascending, but infinite. Note that by Lemma 3.6, for each $g \in M(\mathcal{L})$, the 2-support $[g]_{2}$ is finite.

Let $\mathcal{L}$ contain a copy of the ordinal $\omega$, i.e. there is an ascending sequence $\left(\lambda_{i}\right)_{i \in \omega} \subseteq \Lambda^{+}$. We can successively write $V_{\lambda_{0}}=R u_{0} \oplus C_{0}$ and $V_{\lambda_{i+1}}=V_{\lambda_{i}} \oplus R u_{i} \oplus C_{i}$. Also, let $V=\left(\bigcup_{i \in \omega} V_{\lambda_{i}}\right) \oplus C$. Choose $h \in \operatorname{End}(V)$ with $u_{i+1} h=u_{i}$ and $C_{i} h=0$ for each $i \in w$, and $C h=0$. Then $g=1+h \in \mathcal{G}(\mathcal{L})$ by Proposition 3.4 and $\lambda_{i} \in[g]_{2}$ for each $i \in \omega$. So $g \notin M(\mathcal{L})$.

Now assume that $R$ is a field and suppose that $\mathcal{L}$ is infinite but contains no copy of $\omega$. There is a descending sequence $\left(\lambda_{i}\right)_{i \in \omega} \subseteq \Lambda^{+}$. We may write $V_{\lambda_{n-1}}=V_{\lambda_{n}} \oplus R u_{n} \oplus C_{n}$ and let $U=\bigoplus_{n \in \omega} R u_{n}, C=\bigoplus_{n \in \omega} C_{n}$. Since $R$ is a field, it follows $U \oplus C \oplus D=V$ and we can define $h \in \operatorname{End}(V)$ such that $u_{i} h=u_{i+1}$ for all $i \in \omega$ and $(C \oplus D) h=0$. Then $g=1+h \in \mathcal{G}(\mathcal{L}), \lambda_{i+1} \in[g]_{2}$ for each $i \in \omega$, and $g \notin M(\mathcal{L})$.

Next we show a connection between the present groups and the generalized McLain-groups as investigated in $[4,5]$. We introduce some notation. Let $R$ be a domain and $(S, \leq)$ a linearly ordered set. An $S \times S$-matrix $\left(r_{\alpha \beta}\right)_{\alpha, \beta \in S}$ with $r_{\alpha \beta} \in R$ is called row-finite, if for each $\alpha \in S$ the set $\left\{\beta \in S \mid r_{\alpha \beta} \neq 0\right\}$ is finite, and lower-triangular, if $r_{\alpha \beta}=0$ for all $\alpha, \beta \in S$ with $\alpha<\beta$. Let $\Omega(R, S)$ be the collection of all row-finite lower-triangular $S \times S$-matrices $\left(r_{\alpha \beta}\right)_{\alpha, \beta \in S}$ over $R$ and diagonal $\equiv 1$, i.e., $r_{\alpha \alpha}=1$ for all $\alpha \in S$. With the usual matrix multiplication, $\Omega(R, S)$ 
is a monoid. For $\alpha, \beta \in S$ with $\alpha>\beta$, let $e_{\alpha \beta} \in \Omega(R, S)$ be the matrix with entry 1 at $(\alpha, \beta)$ and 0 elsewhere. Now let $\mathcal{G}(R, S)$ comprise all matrices $A=\left(r_{\alpha \beta}\right) \in \Omega(R, S)$ for which the set $\left.\left\{(\alpha, \beta) \mid r_{\alpha \beta} \neq 0, \alpha>\beta\right)\right\}$ is finite. Then $\mathcal{G}(R, S)$ is a group and generated by the set of all elements $1+a e_{\alpha \beta}(a \in R, \alpha, \beta \in S$ with $\alpha>\beta\}$, cf. [4, Lemma 2.1]. This group $\mathcal{G}(R, S)$ is called the (generalized) McLain-group over $R$ and $S$.

Now let $V$ be a left $R$-module and $\mathcal{L}=\left\{V_{\lambda} \mid \lambda \in \Lambda\right\}$ a direct composition series. For each $\alpha \in \Lambda^{+}$we choose and fix $e_{\alpha} \in V_{\alpha}^{*}$. The set $\left\{e_{\alpha} \mid \alpha \in \Lambda^{+}\right\}$generates a free $R$ module which may be a proper submodule of $V$. We call $\mathcal{L}$ a generating composition series, if $V=\left\langle e_{\alpha} \mid \alpha \in \Lambda^{+}\right\rangle$. Now assume that $\mathcal{L}$ is generating. To each endomorphism $h \in \operatorname{End} V$ stabilizing $\mathcal{L}$ we associate a $\left(\Lambda^{+} \times \Lambda^{+}\right)$-matrix $A_{h}=\left(r_{\alpha \beta}\right)$ over $R$ with respect to the basis $\left\{e_{\alpha} \mid \alpha \in \Lambda^{+}\right\}$as usual, i.e. $e_{\alpha} h=\sum_{i=1}^{m} r_{\alpha \beta_{i}} e_{\beta_{i}}$ with $\alpha, \beta_{1}, \ldots, \beta_{m} \in \Lambda^{+}$and $\alpha>\beta_{1}>\cdots>$ $\beta_{m}$ without loss of generality; we put $r_{\alpha \alpha}=1$ for each $\alpha \in \Lambda^{+}$. Since $h$ is stabilizing, we obtain $A_{h} \in \Omega\left(R, \Lambda^{+}\right)$. Conversely if $A \in \Omega\left(R, \Lambda^{+}\right)$, we obtain a homomorphism $h \in$ End $V$ stabilizing $\mathcal{L}$ with $A=A_{h}$. Since this procedure preserves products, we can identify the monoid $\{g=1+h \mid h \in$ End $V$ stabilies $\mathcal{L}\}$ with $\Omega\left(R, \Lambda^{+}\right)$.

Proposition 3.8. Under the above assumptions, we have $\mathcal{G}\left(R, \Lambda^{+}\right) \subseteq M(\mathcal{L}) \subseteq \mathcal{G}(\mathcal{L}) \subseteq$ $\Omega\left(R, \Lambda^{+}\right)$, and $\mathcal{G}(\mathcal{L})$ is the maximal subgroup of the monoid $\Omega\left(R, \Lambda^{+}\right)$.

Proof. For each $g=1+h \in \mathcal{G}(\mathcal{L})$, the associated matrix $A_{h}$ is invertible in $\Omega\left(R, \Lambda^{+}\right)$. Furthermore, if $A \in \Omega\left(R, \Lambda^{+}\right)$is invertible and $A=A_{h}$ for a stabilizing $h \in \operatorname{End} V$ as above, then $g=1+h \in \operatorname{Aut}_{R} V$, so $g \in \mathcal{G}(\mathcal{L})$. It remains to show that $\mathcal{G}\left(R, \Lambda^{+}\right) \subseteq M(\mathcal{L})$.

Let $a \in R$ and $\alpha, \beta \in \Lambda^{+}$with $\alpha>\beta$. We put $C_{\alpha}=\left\langle e_{\lambda} \mid \lambda>\alpha\right\rangle$. Since $V=\left\langle e_{\lambda} \mid \lambda \in \Lambda^{+}\right\rangle$, we have $V_{\alpha}^{-}=\left\langle e_{\lambda}\right| \lambda\langle\alpha\rangle$, hence $V_{\alpha}=R e_{\alpha} \oplus V_{\alpha}^{-}$and $V=V_{\alpha} \oplus C_{\alpha}$. Now define the McLainelement $g_{\alpha \beta}=1+h_{\alpha \beta}$ such that $e_{\alpha} h_{\alpha \beta}=a e_{\beta}$ and $\left(V_{\alpha}^{-} \oplus C_{\alpha}\right) h_{\alpha \beta}=0$. The choice of $C_{\alpha}$ implies $1+a e_{\alpha \beta}=g_{\alpha \beta} \in M(\mathcal{L})$. Since $\mathcal{G}\left(R, \Lambda^{+}\right)$is generated by the elements $1+a e_{\alpha \beta}(a \in$ $R, \alpha, \beta \in \Lambda^{+}$with $\alpha>\beta$ ), our claim follows.

Proposition 3.9. Under the above assumptions, we have $\mathcal{G}\left(R, \Lambda^{+}\right)=M(\mathcal{L})$ if and only if $\mathcal{L}$ is finite or of order-type $1+\omega^{*}$ or $2+\omega^{*}$. 
Proof. We may assume that $\mathcal{L}$ is infinite. Then $\mathcal{L}$ is not of order-type $1+\omega^{*}$ or $2+\omega^{*}$ iff there are $\alpha, \beta \in \Lambda^{+}$such that $\alpha>\beta$ and $\{\lambda \in \Lambda \mid \lambda>\alpha\}$ is infinite. First assume the latter.

Let $g=1+h_{\alpha \beta}$ be a McLain-element of type $(\alpha, \beta)$. Then by Lemma 3.1, $\lambda \in\left[h_{\alpha \beta}\right]_{1}$ for each $\lambda>\alpha$, so $A_{h_{\alpha \beta}}$ contains 1 in each $\lambda$-row besides at the main diagonal, so $A_{h_{\alpha \beta}}$ is not finite. Hence $g \notin \mathcal{G}\left(R, \Lambda^{+}\right)$.

Now let $\mathcal{L}$ be of order-type $1+\omega^{*}$ or $2+\omega^{*}$. In order to show $\mathcal{G}\left(R, \Lambda^{+}\right)=M(\mathcal{L})$, let $g=1+h_{\alpha \beta} \in M(\mathcal{L})$ be an arbitrary McLain-element obtained from $V=R v_{\alpha} \oplus V_{\alpha}^{-} \oplus C_{\alpha}$ with $\alpha, \beta \in \Lambda^{+}$. Hence $V_{\alpha} \supset V_{\beta} \supset\{0\}=\min (\mathcal{L})$. We claim that $g \in \mathcal{G}\left(R, \Lambda^{+}\right)$. Indeed $V h_{\alpha \beta} \subseteq$ $V_{\alpha}^{-}, V_{\alpha}^{-} h_{\alpha \beta}=\{0\}$, and $V / V_{\alpha}^{-}$and therefore $V h_{\alpha \beta}$ has finite rank. Since $\left\{\lambda \in \Lambda^{+} \mid \lambda \geq \alpha\right\}$ is finite, the matrix $A_{h_{\alpha \beta}}$ has only finitely many non-zero entries outside the diagonal, hence $g \in \mathcal{G}\left(R, \Lambda^{+}\right)$.

\section{Relating $\mathcal{L}$ and $M(\mathcal{L})$.}

In all of this section let $R$ be a ring without zero-divisors and $\mathcal{L}=\left\{V_{\lambda} \mid \lambda \in \Lambda\right\}$ a direct composition series of a left $R$-module $V$. Here we will investigate the relationship between the structure of $\mathcal{L}$ and $M(\mathcal{L})$. First we derive basic properties of McLain-elements.

Lemma 4.1. Let $\alpha>\beta$ and $\alpha>\gamma$. Let $u \in V_{\alpha}^{*}, v \in V_{\beta}^{*}, w \in V_{\gamma}^{*}$, and let $1+h_{\alpha \beta}$ be a McLainelement of type $(\alpha, \beta)$ with $u h_{\alpha \beta}=v$, arising from a decomposition $V=\left(R u \oplus V_{\alpha}^{-}\right) \oplus C$ as above in Definition 3.5. Let $h: V \rightarrow V$ be a homomorphism with $v h=w$. Then $1+h_{\alpha \beta} h$ is a McLain-element of type $(\alpha, \gamma)$ arising from the same decomposition of $V$ as $h_{\alpha \beta}$, and $u h_{\alpha \beta} h=w$.

Proof. Trivial.

Lemma 4.2. Let $\alpha>\beta>\gamma$, and let $1+h_{\alpha \beta}, 1+h_{\beta \gamma}$ be McLain-elements of type $(\alpha, \beta)$ resp. $(\beta, \gamma)$. Then $1+h_{\alpha \beta} h_{\beta \gamma}$ is a McLain-element of type $(\alpha, \gamma)$, arising from any decomposition of $V$ as for $h_{\alpha \beta}$.

Proof. Let $u \in V_{\alpha}^{*}$ with $V=\left(R u \oplus V_{\alpha}^{-}\right) \oplus C$ and $v=u h_{\alpha \beta} \in V_{\beta}^{*}$. Let $v^{\prime} \in V_{\beta}^{*}$ with $V_{\beta}=R v^{\prime} \oplus V_{\beta}^{-}$and $v^{\prime} h_{\beta \gamma}=w \in V_{\gamma}^{*}$. Then $v=r v^{\prime}+w^{\prime}$ for some $0 \neq r \in R, w^{\prime} \in V_{\beta}^{-}$. So $v h_{\beta \gamma}=r v^{\prime} h_{\beta \gamma}=r w \in V_{\gamma}^{*}$. Lemma 4.1 implies the claim. 
Lemma 4.3. Let $\alpha>\beta, \gamma>\delta$ and either

(a) $\gamma>\beta$ or

(b) $\beta>\gamma, V=\left(R u \oplus V_{\alpha}^{-}\right) \oplus C$ a decomposition for $h_{\alpha \beta}$ with $u h_{\alpha \beta}=v \in V_{\beta}^{*}$ and $V=$ $\left(R w \oplus V_{\gamma}^{-}\right) \oplus C^{\prime}$ a decomposition for $h_{\gamma \delta}$ with $v \in C^{\prime}$.

Then $h_{\alpha \beta} h_{\gamma \delta}=0$.

Proof. By $V_{\beta} \subseteq V_{\gamma}^{-}$resp. $v \in C^{\prime}$ and $C^{\prime} h_{\gamma \delta}=0$.

In all of this section let $U$ be a group such that $M(\mathcal{L}) \subseteq U \subseteq \mathcal{G}(\mathcal{L})$. The following 'commutator lemma' will be very important for us.

Lemma 4.4. Let $g=1+a \in U$ with $g^{-1}=1+a^{*}$. Let $\zeta>\delta$ in $\Lambda^{+}$and $e=1+h_{\zeta \delta}$.

Then the commutator $c=[g, e]=1-a^{*} h_{\zeta \delta}-\left(1+a^{*}\right) h_{\zeta \delta} a$, hence

$[e, g]=[g, e]^{-1}=1+a^{*} h_{\zeta \delta}+\left(1+a^{*}\right) h_{\zeta \delta} a$. If also $\gamma>\xi \geq \zeta$ in $\Lambda^{+}$and $f=1+h_{\gamma \xi}$, then $[c, f]=1+h_{\gamma \xi} a^{*} h_{\zeta \delta} g+h_{\gamma \xi} h_{\zeta \delta} a$.

Proof. First we recall that $a+a^{*}+a a^{*}=a^{*}+a+a^{*} a=0$. Hence

$$
\begin{aligned}
c & =[g, e]=g^{-1} e^{-1} g e=\left(1+a^{*}\right)\left(1-h_{\zeta \delta}\right)(1+a)\left(1+h_{\zeta \delta}\right) \\
& =1-h_{\zeta \delta} a+a h_{\zeta \delta}-a^{*} h_{\zeta \delta} a+a^{*} a h_{\zeta \delta} \\
& =1-h_{\zeta \delta} a-a^{*} h_{\zeta \delta} a-a^{*} h_{\zeta \delta}=1+k .
\end{aligned}
$$

Using $k^{2}=0$, so $k^{*}=-k$, the formula for $[e, g]$ is clear. Now let $\gamma>\xi \geq \zeta$ and $f=1+h_{\gamma \xi}$. The formula for $[g, e]$ implies

$$
\begin{aligned}
{[c, f] } & =1+k h_{\gamma \xi}-(1-k) h_{\gamma \xi} k \\
& =1-h_{\gamma \xi} k \\
& =1+h_{\gamma \xi} h_{\zeta \delta} a+h_{\gamma \xi} a^{*} h_{\zeta \delta} a+h_{\gamma \xi} a^{*} h_{\zeta \delta},
\end{aligned}
$$

as claimed.

If $A \subseteq \Lambda^{+}$we let $A \uparrow=\left\{\lambda \in \Lambda^{+} \mid \lambda>\mu\right.$ for some $\left.\mu \in A\right\}$ and we define $A \downarrow$ dually. Next we consider particular pairs of subsets of $\Lambda^{+}$which resemble Dedekind cuts in linear orderings and which will be used to define particular normal subgroups of $U$.

Definition 4.5. Let $A, B \subseteq \Lambda^{+}$. The pair $(A, B)$ is a couple if the following conditions hold: 
(1) $A \neq \emptyset \neq B$,

(2) $A>B$,

(3) $A$ is closed upwards, i.e. $A=A \uparrow$, and $B$ is closed downwards, i.e. $B=B \downarrow$.

We write $(A, B) \subseteq\left(A^{\prime}, B^{\prime}\right)$ if $A \subseteq A^{\prime}$ and $B \subseteq B^{\prime}$. Let $N_{A, B}=\{g \in U \mid[g] \subseteq A \times B\}$, which we call an order-theoretic normal subgroup of $U$.

Lemma 4.6. (a) $N_{A, B}$ is an abelian normal subgroup of $U$.

(b) $(A, B) \subseteq\left(A^{\prime}, B^{\prime}\right)$ iff $N_{A, B} \subseteq N_{A^{\prime}, B^{\prime}}$

Proof. (a) Let $g=1+a$ and $h=1+b$ be elements in $N_{A, B}$. Then $g h=1+a+b+a b$ and $h g=1+a+b+b a$. We will show that $a b=b a=0$. If $a b \neq 0$, then there is $(\alpha, \gamma) \in[a b]$. Hence there is $\beta$ with $(\alpha, \beta) \in[a]$ and $(\beta, \gamma) \in[b]$, so $\beta \in A \cap B$, a contradiction. Thus $g$ and $h$ commute. Moreover, $g h \in N_{A, B}$ and $a^{2}=0$, so $g^{-1}=1-a \in N_{A, B}$.

Now let $k=1+u \in U$ with $k^{-1}=1+u^{*}$. Then

$$
\begin{aligned}
g^{k} & =\left(1+u^{*}\right)(1+a)(1+u)=1+a+u+u^{*}+u^{*} a+a u+u^{*} u+u^{*} a u \\
& =1+a+u^{*} a+a u+u^{*} a u .
\end{aligned}
$$

Then $\left[u^{*} a\right],[a u],\left[u^{*} a u\right] \subseteq A \times B$, so $g^{k} \in N_{A, B}$. Hence $N_{A, B}$ is normal in $U$.

(b) Let $(A, B) \subseteq\left(A^{\prime}, B^{\prime}\right)$ and let $g=1+a \in N_{A, B}$. Then $[g] \subseteq A \times B \subseteq A^{\prime} \times B^{\prime}$, so $g \in N_{A^{\prime}, B^{\prime}}$. Conversely, suppose $N_{A, B} \subseteq N_{A^{\prime}, B^{\prime}}$. Choose $(\alpha, \beta) \in A \times B$. The McLain-element $g=1+h_{\alpha \beta}$ belongs to $U$, so it satisfies $g \in N_{A, B} \subseteq N_{A^{\prime}, B^{\prime}}$. Hence $(\alpha, \beta) \in[g] \subseteq A^{\prime} \times B^{\prime}$, showing $(A, B) \subseteq\left(A^{\prime}, B^{\prime}\right)$.

Subsequently we denote by $\top$ the greatest element of $\Lambda^{+}$and by $\perp$ the smallest element of $\Lambda^{+}$, provided they exist.

Lemma 4.7. Let $N$ be an abelian subgroup of $U$ with normalizer containing $M(\mathcal{L})$. Suppose $g=1+a, h=1+b \in N$ and $\alpha>\beta \geq \gamma>\delta$ in $\Lambda^{+}$with $(\alpha, \beta) \in[g]$ and $(\gamma, \delta) \in[h]$. Then $\alpha=\top, \delta=\perp$, and $\beta=\gamma$ or $\beta \succ \gamma$. Consequently, if $k \in N$, then [ $k]$ can only contain the pairs $(\mu, \nu)$ if $\mu>\beta \geq \gamma>\nu$ and possibly $(\top, \beta)$ or $(\gamma, \perp)$, possibly $(\top, \gamma)$ or $(\beta, \perp)$ if $\beta \succ \gamma$, and possibly either $(\top, \nu)$ if $\nu \succ \beta=\gamma$, or $(\mu, \perp)$ if $\beta=\gamma \succ \mu$ (but not both). 
Proof. Let $g^{-1}=1+a^{*}$ and $h^{-1}=1+b^{*}$.

Case 1. Let $\beta>\gamma$. Suppose there is $\delta^{\prime} \in \Lambda^{+}$with $\delta>\delta^{\prime}$. We can choose $u \in V_{\alpha}^{*}$ such that $u a \in V_{\beta}^{*}$. Since $[b]=\left[b^{*}\right]$ by Lemma 3.2 , there is $v \in V_{\gamma}^{*}$ such that $v b^{*} \in V_{\delta}^{*}$. Choose $u^{\prime} \in V_{\beta}^{*}$, $v^{\prime} \in V_{\delta}^{*}$ and $w \in V_{\delta^{\prime}}^{*}$ with $V_{\beta}=R u^{\prime} \oplus V_{\beta}^{-}$and $V_{\delta}=R v^{\prime} \oplus V_{\delta}^{-}$, and write $V=V_{\beta} \oplus C=V_{\delta} \oplus C^{\prime}$. Next let $f=1+h_{\beta \gamma}$ and $e=1+h_{\delta \delta^{\prime}}$ be the McLain-elements arising from $u^{\prime}, v, C$ respectively $v^{\prime}, w, C^{\prime}$. By $\beta>\gamma>\delta>\delta^{\prime}$ and Lemma 4.4, we have

$$
N \ni h^{\prime}=[[h, e], f]=1+h_{\beta \gamma} b^{*} h_{\delta \delta^{\prime}} h+h_{\beta \gamma} h_{\delta \delta^{\prime}} b=1+b^{\prime} .
$$

We have $u a=r_{u} u^{\prime}+u^{\prime \prime}$ and $v b^{*}=r_{v} v^{\prime}+v^{\prime \prime}$ with $0 \neq r_{u}, r_{v} \in R$ and $u^{\prime \prime} \in V_{\beta}^{-}, v^{\prime \prime} \in V_{\delta}^{-}$. Then $u a b^{\prime}=r_{u} u^{\prime} b^{\prime}=r_{u} r_{v}(w+w b)+r_{u} v h_{\delta \delta^{\prime}} b \in r_{u} r_{v} w+V_{\delta^{\prime}}^{-} \subseteq V_{\delta^{\prime}}^{*}$. But $u b^{\prime} \in V_{\delta^{\prime}}$ and $u b^{\prime} a \in V_{\delta^{\prime}}^{-}$. Hence $a$ and $b^{\prime}$ do not commute, a contradiction. Hence $\delta=\perp$.

Case 2. Let $\beta=\gamma$. Suppose there is $\delta^{\prime} \in \Lambda^{+}$with $\delta>\delta^{\prime}$. Again $[b]=\left[b^{*}\right]$.

First we show that whenever $w \in V_{\beta}^{-}$then $w b^{*} \in V_{\delta^{\prime}}$. Indeed, otherwise there are $\beta>\beta^{\prime}>\gamma^{\prime}>\delta^{\prime}$ and $w \in V_{\beta^{\prime}}^{*}$ with $w b^{*} \in V_{\gamma^{\prime}}^{*}$. Then $\left(\beta^{\prime}, \gamma^{\prime}\right) \in\left[b^{*}\right]=[h]$ and we get a contradiction by Case 1 .

Now we choose $u^{\prime} \in V_{\beta}^{*}$ with $V_{\beta}=R u^{\prime} \oplus V_{\beta}^{-}$. We claim that $u^{\prime} b^{*} \in V_{\delta}^{*}$. Indeed, by $(\beta, \delta) \in[b]=\left[b^{*}\right]$ there is $v \in V_{\beta}^{*}$ with $v b^{*} \in V_{\delta}^{*}$. Write $v=y u^{\prime}+v^{\prime \prime}$ with $0 \neq y \in R$ and $v^{\prime \prime} \in V_{\beta}^{-}$. Then $v b^{*}=y u^{\prime} b^{*}+v^{\prime \prime} b^{*} \in V_{\delta}^{*}$ and $v^{\prime \prime} b^{*} \in V_{\delta^{\prime}}$ as shown above. Hence $y u^{\prime} b^{*} \in V_{\delta}^{*}$ and our claim follows.

We choose $v^{\prime} \in V_{\delta}^{*}$ and $w^{\prime} \in V_{\delta^{\prime}}^{*}$ with $V_{\delta}=R v^{\prime} \oplus V_{\delta}^{-}$, and write $V=V_{\delta} \oplus C$. Let $e=1+h_{\delta \delta^{\prime}}$ be the McLain-element arising from $v^{\prime}, w^{\prime}, C$. By Lemma 4.4 we have

$$
N \ni h^{\prime}=[h, e]=1-b^{*} h_{\delta \delta^{\prime}}-\left(1+b^{*}\right) h_{\delta \delta^{\prime}} b=1+b^{\prime} .
$$

Now choose $u \in V_{\alpha}^{*}$ with $u a \in V_{\beta}^{*}$. Then $u a=x u^{\prime}+u^{\prime \prime}$ for some $0 \neq x \in R$ and $u^{\prime \prime} \in V_{\beta}^{-}$. By our first claim, we have $u^{\prime \prime} b^{*} \in V_{\delta^{\prime}}$, and hence $u^{\prime \prime} b^{\prime} \in V_{\delta^{\prime}}^{-}$. By our second claim, $u^{\prime} b^{*}=z v^{\prime}+w^{\prime \prime}$ for some $0 \neq z \in R$ and $w^{\prime \prime} \in V_{\delta}^{-}$. So $u^{\prime} b^{*} h_{\delta \delta^{\prime}}=z w^{\prime}$, hence $u^{\prime} b^{\prime} \in-z w+V_{\delta^{\prime}}^{-}$. Thus $u a b^{\prime}=x u^{\prime} b^{\prime}+u^{\prime \prime} b^{\prime} \in-x z w^{\prime}+V_{\delta^{\prime}}^{-} \subseteq V_{\delta^{\prime}}^{*}$. But $u b^{\prime} \in V_{\delta^{\prime}}$ and $u b^{\prime} a \in V_{\delta^{\prime}}^{-}$, so $a$ and $b^{\prime}$ do not commute, a contradiction. Hence $\delta=\perp$.

Case 3. Let $\beta>\gamma$. Suppose there is $\alpha^{\prime} \in \Lambda^{+}$with $\alpha^{\prime}>\alpha$. Since $[a]=\left[a^{*}\right]$, we can choose $u \in V_{\alpha}^{*}$ with $u a^{*} \in V_{\beta}^{*}$ and $v \in V_{\gamma}^{*}$ with $v^{\prime}=v b \in V_{\delta}^{*}$. Write $V_{\beta}=R u^{\prime} \oplus V_{\beta}^{-}$. Then $u a^{*}=r_{u} u^{\prime}+u^{\prime \prime}$ with $0 \neq r_{u} \in R$ and $u^{\prime \prime} \in V_{\beta}^{-}$. Since $V$ is torsionless, we can choose a decomposition $V=\left(R u^{\prime} \oplus V_{\beta}^{-}\right) \oplus C^{\prime}$ with $u \in C^{\prime}$. Next, choose McLain-elements $e=1+h_{\beta \gamma}$ 
arising from $u^{\prime}, v, C^{\prime}$ and $f=1+h_{\alpha^{\prime} \alpha}$ with $w h_{\alpha^{\prime} \alpha}=u$ for some $w \in V_{\alpha^{\prime}}^{*}$. Then $\alpha^{\prime}>\alpha>\beta>\gamma$ and $h_{\alpha^{\prime} \alpha} h_{\beta \gamma}=0$ by Lemma $4.3(\mathrm{~b})$. By Lemma 4.4 we get

$$
N \ni g^{\prime}=[[g, e], f]=1+h_{\alpha^{\prime} \alpha} a^{*} h_{\beta \gamma} g+h_{\alpha^{\prime} \alpha} h_{\beta \gamma} a=1+h_{\alpha^{\prime} \alpha} a^{*} h_{\beta \gamma}(1+a)=1+a^{\prime} .
$$

Note that $a b=b a$, so $w a^{\prime} b=r_{u}(v+v a) b=r_{u}\left(v^{\prime}+v b a\right)=r_{u}\left(v^{\prime}+v^{\prime} a\right) \in V_{\delta}^{*}$ as $v^{\prime} a \in V_{\delta}^{-}$. But $w b \in V_{\alpha^{\prime}}^{-}$, so $w b a^{\prime}=0$ and $b a^{\prime} \neq a^{\prime} b$, a contradiction. So $\alpha=\top$.

Case 4. Let $\beta=\gamma$. Suppose there is $\alpha^{\prime} \in \Lambda^{+}$with $\alpha^{\prime}>\alpha$. We first show that $v b=0$ for each $v \in V_{\beta}^{-}$. Indeed, otherwise there is $\left(\beta^{\prime}, \delta^{\prime}\right) \in[b]$ with $\alpha>\beta>\beta^{\prime}>\delta^{\prime}$, so Case 3 yields $\alpha=\top$.

Choose $u \in V_{\alpha}^{*}$ with $u a \in V_{\beta}^{*}$, and $v \in V_{\beta}^{*}$ with $V_{\beta}=R v \oplus V_{\beta}^{-}$. Choose $w \in V_{\beta}^{*}$ with $w b \in V_{\delta}^{*}$. Then $w=y v+w^{\prime}$ for some $0 \neq y \in R$ and $w^{\prime} \in V_{\beta}^{-}$. But $w^{\prime} b=0$, so $y v b=w b \in V_{\delta}^{*}$ showing $v b \in V_{\delta}^{*}$.

Now $u a=x v+v^{\prime}$ for some $0 \neq x \in R$ and $v^{\prime} \in V_{\beta}^{-}$. Again $v^{\prime} b=0$, so $u a b=x v b \in V_{\delta}^{*}$.

Now choose $u^{\prime} \in V_{\alpha^{\prime}}^{*}$ and a McLain-element $e=1+h_{\alpha^{\prime} \alpha}$ with $u^{\prime} h_{\alpha^{\prime} \alpha}=u$. By Lemma 4.4 we obtain

$$
N \ni g^{\prime}=[g, e]=1-a^{*} h_{\alpha^{\prime} \alpha}-\left(1+a^{*}\right) h_{\alpha^{\prime} \alpha} a=1+a^{\prime} .
$$

Then $u^{\prime} b a^{\prime}=0$, but $u^{\prime} a^{\prime} b=-u^{\prime} h_{\alpha^{\prime} \alpha} a b=-u a b \in V_{\delta}^{*}$, so $b a^{\prime} \neq a^{\prime} b$, a contradiction. Hence $\alpha=\top$.

Case 5. There is $\eta \in \Lambda^{+}$with $\beta>\eta>\gamma$. Again, since $[a]=\left[a^{*}\right]$, we can choose $u \in V_{\alpha}^{*}$ with $u a^{*} \in V_{\beta}^{*}$ and $v \in V_{\gamma}^{*}$ with $v b \in V_{\delta}^{*}$. Decompose $V_{\beta}=R u^{\prime} \oplus V_{\beta}^{-}$and $V_{\eta}=R w \oplus V_{\eta}^{-}$. Write $u a^{*}=r_{u} u^{\prime}+u^{\prime \prime}$ with $0 \neq r_{u} \in R$ and $u^{\prime \prime} \in V_{\beta}^{-}$. Let $e=1+h_{\beta \eta}$ and $e^{\prime}=1+h_{\eta \gamma}$ be McLain-elements such that $u^{\prime} h_{\beta \eta}=w$ and $w h_{\eta \gamma}=v$. By Lemma 4.4 then

$$
\begin{aligned}
N \ni[g, e] & =1-a^{*} h_{\beta \eta}-\left(1+a^{*}\right) h_{\beta \eta} a=1+c \text { and } \\
N \ni\left[h, e^{\prime}\right] & =1-b^{*} h_{\eta \gamma}-\left(1+b^{*}\right) h_{\eta \gamma} b=1+d .
\end{aligned}
$$

Then $u c d=u a^{*} h_{\beta \eta} h_{\eta \gamma} b=r_{u} v b \in V_{\delta}^{*}$ but $u d c=0$, contradicting that $N$ is abelian. Hence $\beta>\gamma$ implies $\beta \succ \gamma$.

Finally, the last statement of the lemma is immediate by the preceding one.

Trivially we have $0, V \in \mathcal{L}$. We put $\mathcal{L}^{*}=\mathcal{L} \backslash\{0, V\}$. 
Theorem 4.8. Let $\mathcal{L}^{*}$ contain no maximal or no minimal element. Then each abelian normal subgroup $N$ of $U$ is contained in an order-theoretic normal subgroup $N_{A, B}$.

Proof. If $N \neq 1$, let $A=\bigcup_{g \in N}[g]_{1}$ and $B=\bigcup_{h \in N}[h]_{2}$. By Lemma 3.1, $A$ is closed upwards. Next we show that $B=B \downarrow$. Let $(\alpha, \beta) \in[g]$ for some $g=1+a \in N$, and let $\beta>\gamma$. Choose $u \in V_{\alpha}^{*}$ and $v \in V_{\beta}^{*}$ with $u a^{*}=v$. Decompose $V_{\beta}=R v^{\prime} \oplus V_{\beta}^{-}$. Then $v=r v^{\prime}+v^{\prime \prime}$ for some $0 \neq r \in R$ and $v^{\prime \prime} \in V_{\beta}^{-}$. Next choose a McLain-element $e=1+h_{\beta \gamma}$ with $v^{\prime} h_{\beta \gamma}=w \in V_{\gamma}^{*}$. Then $N \ni c=[g, e]=1+d$, and Lemma 4.4 shows that $u d \in-r w+V_{\gamma}^{-}$, so $(\alpha, \gamma) \in[c]$ and $\gamma \in B$.

By Lemma 4.7 there are no $g, h \in N$ with $(\alpha, \beta) \in[g]$, and $(\gamma, \delta) \in[h]$ such that $\alpha>\beta \geq \gamma>\delta$. It follows that $A>B$. Hence $(A, B)$ is a couple, since $N \neq 1$, and $N \subseteq N_{A, B}$ by construction.

A chain $(C, \leq)$ is called Dedekind-complete, if for any non-empty subset $A \subseteq C$ which has an upper bound in $C$ there exists the supremum (= least upper bound) $\sup A$ in $(C, \leq)$; equivalently, any non-empty lower bounded subset has an infimum in $C$. Clearly, since $\mathcal{L}$ is closed under unions and intersections, the chain $(\mathcal{L}, \subseteq)$ and thus also $(\Lambda, \leq)$ is Dedekindcomplete.

For any $\lambda \in \Lambda$, let $(\infty, \lambda)=\{\gamma \in \Lambda \mid \gamma>\lambda\}$ and $(\infty, \lambda]=\{\gamma \in \Lambda \mid \gamma \geq \lambda\}$. Similarly, the intervals $(\lambda,-\infty)$ and $[\lambda,-\infty)$ are defined.

For $\lambda \in \Lambda$ let $N_{\lambda}=\left\{g \in U \mid[g]_{1}>\lambda \geq[g]_{2}\right\}$. Hence $N_{\lambda}$ is an order-theoretic normal subgroup of $U$ by Lemma 4.6(a).

Let $(A, B)$ be a maximal couple. Since $\Lambda$ is Dedekind-complete, either $A=(\infty, \lambda) \cap \Lambda^{+}$ and $B=[\lambda,-\infty) \cap \Lambda^{+}$, or $A=(\infty, \lambda] \cap \Lambda^{+}$and $B=(\lambda,-\infty) \cap \Lambda^{+}$where $\lambda \in \Lambda$. If $\lambda$ has a predecessor $\eta$, clearly $(\infty, \lambda] \cap \Lambda^{+}=(\infty, \eta) \cap \Lambda^{+}$and $(\lambda,-\infty) \cap \Lambda^{+}=[\eta,-\infty) \cap \Lambda^{+}$. If $\lambda$ has no predecessor, we have $\lambda \notin \Lambda^{+}$, hence $(\infty, \lambda] \cap \Lambda^{+}=(\infty, \lambda) \cap \Lambda^{+}$and $(\lambda,-\infty) \cap \Lambda^{+}=$ $[\lambda,-\infty) \cap \Lambda^{+}$. Hence we can always write $A=(\infty, \lambda) \cap \Lambda^{+}$and $B=[\lambda,-\infty) \cap \Lambda^{+}$for some $\lambda \in \Lambda$, so $N_{A, B}=N_{\lambda}$.

As an immediate consequence of Theorem 4.8 we obtain a group-theoretic characterization of the order-theoretically defined normal subgroups $N_{\lambda}$.

Corollary 4.9. Let $\mathcal{L}^{*}$ contain no maximal or no minimal element. Then the maximal abelian normal subgroups of $U$ are precisely the groups $N_{\lambda}(\lambda \in \Lambda)$. 
Proof. By Theorem 4.8, all maximal abelian normal subgroups of $U$ are of this form. Conversely, to show that each $N_{\lambda}$ is a maximal abelian normal subgroup, apply again Theorem 4.8 and observe that if $N_{\lambda} \subseteq N_{\mu}$, then $\lambda=\mu$ by Lemma 4.6 .

The following tool will enable us to recover the order structure of $(\Lambda, \leq)$ via the groups $N_{\lambda}$.

Lemma 4.10. Let $\mu, \nu, \lambda \in \Lambda$. Then $N_{\mu} \cap N_{\nu} \subseteq N_{\lambda}$ if and only if $\lambda$ lies between $\mu$ and $\nu$, i.e., either $\mu \geq \lambda \geq \nu$ or $\nu \geq \lambda \geq \mu$.

Proof. We may assume that $\mu \geq \nu$. Hence $N_{\mu} \cap N_{\nu}=N_{(\infty, \mu),[\nu,-\infty)}$. By Lemma 4.6(b), we have $N_{(\infty, \mu),[\nu,-\infty)} \subseteq N_{\lambda}$ iff $\mu \geq \lambda \geq \nu$.

Now we obtain:

Theorem 4.11. Let $\mathcal{L}_{1}, \mathcal{L}_{2}$ be two direct composition series such that $\mathcal{L}_{1}^{*}, \mathcal{L}_{2}^{*}$ each have either no maximal or no minimal element. Let $M\left(\mathcal{L}_{i}\right) \subseteq U_{i} \subseteq \mathcal{G}\left(\mathcal{L}_{i}\right)$ for $i=1,2$, and assume that $U_{1} \cong U_{2}$. Then the chains $\left(\mathcal{L}_{1}, \subseteq\right)$ and $\left(\mathcal{L}_{2}, \subseteq\right)$ are either isomorphic or anti-isomorphic.

Proof. Let $\varphi: U_{1} \rightarrow U_{2}$ be the given isomorphism. Then $\varphi$ maps the maximal abelian normal subgroups of $U_{1}$ bijectively onto those of $U_{2}$. Hence, by Corollary $4.9, \varphi$ induces a bijection $\psi: \Lambda_{1} \rightarrow \Lambda_{2}$ satisfying $N_{\lambda}^{\varphi}=N_{\lambda \psi}$ for each $\lambda \in \Lambda_{1}$. By Lemma 4.10, $\psi$ and $\psi^{-1}$ preserve the induced betweenness relations of the chains $\left(\Lambda_{1}, \leq\right),\left(\Lambda_{2}, \leq\right)$. Thus, $\psi:\left(\Lambda_{1}, \leq\right.$ )$\rightarrow\left(\Lambda_{2}, \leq\right)$ is either an order-isomorphism or anti-isomorphism.

\section{Bounded composition series}

We call a chain $(C, \leq)$ bounded, if $(C, \leq)$ contains both a greatest and a smallest element, denoted by $\max C$ respectively $\min C$. Now we investigate the case that $\Lambda^{+}$is bounded. We write $\top=\max \Lambda^{+}, \perp=\min \Lambda^{+}$. Recall that $V_{\perp}^{-}=0$ and $V_{\top}=V$. For each $\lambda \in \Lambda^{+}$we fix decompositions $V_{\lambda}=R v_{\lambda} \oplus V_{\lambda}^{-}$with $v_{\lambda} \in V_{\lambda}^{*}$. Hence for each $\mu>\nu$ in $\Lambda^{+}$any McLainelement $h=1+h_{\mu \nu}$ determines a unique ring element $r_{\mu \nu} \in R$ defined by $v_{\mu} h_{\mu \nu} \equiv r_{\mu \nu} v_{\nu}$ $\bmod V_{\nu}^{-}$. We call $r_{\mu \nu}$ the ring element associated with $h_{\mu \nu}$. Conversely, for each $r \in R$ we have an associated McLain-element of type $(\mu, \nu)$ given by $h^{r}=1+h_{\mu \nu}^{r}$ where $v_{\mu} h_{\mu \nu}^{r}=r v_{\nu}$ 
and $\left(V_{\mu}^{-} \oplus C_{\mu}\right) h_{\mu \nu}^{r}=0$. Furthermore, for each $\beta, \gamma \in \Lambda^{+}$with $\beta \succ \gamma$, we have $V=V_{\gamma} \oplus C_{\gamma}$ and $v_{\beta}=r_{\beta} v_{\gamma}+c$ for some uniquely determined $r_{\beta} \in R$ and $c \in V_{\gamma}^{-} \oplus C_{\gamma}$. Formally we put $r_{\beta}=1$ if $\beta=\gamma$.

Lemma 5.1. Observe that $V h_{\mu \nu}^{r}=R r v_{\nu}$. Whenever $\beta \succ \gamma$ and $t \in R$, then $v_{\beta} h_{\gamma \perp}^{t}=$ $r_{\beta} v_{\gamma} h_{\gamma \perp}^{t}=r_{\beta} t v_{\perp}=v_{\beta} h_{\beta \perp}^{r_{\beta} t}$, so $v_{\beta}\left(h_{\gamma \perp}^{t}-h_{\beta \perp}^{r_{\beta} t}\right)=0$.

Next show that elements of the type described in Lemma 4.7 can be written in a particular standard form.

Lemma 5.2. Let $\top>\beta \geq \gamma>\perp$ in $\Lambda^{+}$such that either $\beta \succ \gamma$ or $\beta=\gamma$, and $g \in \mathcal{G}(\mathcal{L})$. Assume that $[g]$ contains at most the pairs $(\top, \beta),(\top, \gamma),(\beta, \perp),(\gamma, \perp)$ and $(\mu, \nu)$ with $\mu>$ $\beta \geq \gamma>\nu$. Then $g$ can be written in the form

$$
g=1+h_{\top \beta}^{p}+h_{\top \gamma}^{q}+h_{\beta \perp}^{s}+h_{\gamma \perp}^{t}-h_{\beta \perp}^{r_{\beta} t}+a^{\prime}
$$

with $p, q, s, t \in R, q=t=0$ if $\beta=\gamma$, and $\left[a^{\prime}\right] \subseteq[\top, \beta) \times(\gamma, \perp]$. Moreover, $p, q, s, t$ and $a^{\prime}$ as above are unique. We have $p \neq 0$ iff $(\top, \beta) \in[g]$, and if $\beta \succ \gamma$, then $t \neq 0$ iff $(\gamma, \perp) \in[g]$.

Proof. Write $g=1+a$. We have $v_{\top} a=p v_{\beta}+v^{\prime}$ for some $p \in R$ and $v^{\prime} \in V_{\beta}^{-} \subseteq V_{\gamma}$. Then $v^{\prime}=q v_{\gamma}+v^{\prime \prime}$ with $q \in R$ and $v^{\prime \prime} \in V_{\gamma}^{-}$. If $\beta=\gamma$, here clearly $q=0$. Then $v_{\top} a=v_{\top}\left(h_{\top \beta}^{p}+h_{\top \gamma}^{q}\right)+v^{\prime \prime}$. Furthermore, since $v_{\beta} a, v_{\gamma} a \in V_{\perp}$ and $V_{\perp}^{-}=\{0\}$, we get $v_{\beta} a=v_{\beta} h_{\beta \perp}^{s}$ and $v_{\gamma} a=v_{\gamma} h_{\gamma \perp}^{t}$ for some $s, t \in R$. In case $\beta=\gamma$, here we put $t=0$. Put $a^{\prime}=a-\left(h_{\top \beta}^{p}+h_{\top \gamma}^{q}+h_{\beta \perp}^{s}+h_{\gamma \perp}^{t}-h_{\beta \perp}^{r_{\beta} t}\right)$. We have to show that $\left[a^{\prime}\right] \subseteq[\top, \beta) \times(\gamma, \perp]$. First let $v \in V_{\top}$. Then $v=z v_{\top}+w$ for some $z \in R$ and $w \in V_{\top}^{-}$. By definition of $a^{\prime}$, we have $v_{\top} a^{\prime}=v^{\prime \prime}-v_{\top}\left(h_{\beta \perp}^{s}+h_{\gamma \perp}^{t}-h_{\beta \perp}^{r_{\beta} t}\right) \in V_{\gamma}^{-}$and similarly $w a^{\prime} \in w a+V_{\perp} \subseteq V_{\gamma}^{-}$by assumption on $[g]$, so $v a^{\prime} \in V_{\gamma}^{-}$.

If $w \in V_{\beta}$, then $w=x v_{\beta}+y v_{\gamma}+w^{\prime}$ for some $x, y \in R$ and $w^{\prime} \in V_{\gamma}^{-}$, letting $y=0$ if $\beta=\gamma$. Then $w^{\prime} a^{\prime}=w^{\prime} a=0$ by assumption on $[a]$. Furthermore, $v_{\gamma} a^{\prime}=v_{\gamma} a-v_{\gamma} h_{\gamma \perp}^{t}=0$ and $v_{\beta} a^{\prime}=v_{\beta} a-v_{\beta}\left(h_{\beta \perp}^{s}+h_{\gamma \perp}^{t}-h_{\beta \perp}^{r_{\beta} t}\right)=0$ by Lemma 5.1. Hence $w a^{\prime}=0$ and our claim about $\left[a^{\prime}\right]$ follows. Finally, the uniqueness of $p, q, s, t$ and $a^{\prime}$ and the properties of $p$ and $t$ are easy by considering the action of $a$ on $v_{\top}, v_{\beta}$ and $v_{\gamma}$.

Now we consider the commutation behavior of elements having the standard form just described. 
Lemma 5.3. Let $\top>\beta \geq \gamma>\perp$ such that either $\beta \succ \gamma$ or $\beta=\gamma$, and let $g, h \in \mathcal{G}(\mathcal{L})$. Assume that $[g],[h]$ contain at most the pairs $(\top, \beta),(\top, \gamma),(\beta, \perp),(\gamma, \perp)$ and $(\mu, \nu)$ with $\mu>\beta \geq \gamma>\nu$. Choose $p, q, s, t \in R$ as in Lemma 5.2 for $g$ and $p^{\prime}, q^{\prime}, s^{\prime}, t^{\prime} \in R$ for $h$. Then

(a) $g$ and $h$ commute iff $p s^{\prime}+q t^{\prime}=p^{\prime} s+q^{\prime} t$.

(b) $h$ commutes with all $M(\mathcal{L})$-conjugates $g^{m}$ of $g(m \in M(\mathcal{L}))$ iff $p s^{\prime}+q t^{\prime}=p^{\prime} s+q^{\prime} t$ and $p x t^{\prime}=-p^{\prime} x t$ for each $x \in R$.

(c) Assume $(\top, \beta),(\gamma, \perp) \in[g]$ and $\beta \succ \gamma$. Then $g$ commutes with all its $M(\mathcal{L})$-conjugates iff $\operatorname{char}(R)=2$.

Proof. (a),(b) By Lemma 5.2, write $g=1+a+a^{\prime}$ and $h=1+b+b^{\prime}$ with

(i) $a=h_{\top \beta}^{p}+h_{\top \gamma}^{q}+h_{\beta \perp}^{s}+h_{\gamma \perp}^{t}-h_{\beta \perp}^{r_{\beta} t}$,

(ii) $b=h_{\top \beta}^{p^{\prime}}+h_{\top \gamma}^{q^{\prime}}+h_{\beta \perp}^{s^{\prime}}+h_{\gamma \perp}^{t^{\prime}}-h_{\beta \perp}^{r_{\beta} t^{\prime}}$,

(iii) $\left[a^{\prime}\right],\left[b^{\prime}\right] \subseteq[\top, \beta) \times(\gamma, \perp]$,

(iv) $p, q, s, t, p^{\prime}, q^{\prime}, s^{\prime}, t^{\prime} \in R$ and $q=q^{\prime}=t=t^{\prime}=0$ if $\beta=\gamma$.

Then $a a^{\prime}=0$, so $g=1+a+a^{\prime}=(1+a)\left(1+a^{\prime}\right)$ and similarly $h=(1+b)\left(1+b^{\prime}\right)$. Let $m=1+c \in M(\mathcal{L})$ and $m^{-1}=1+c^{*}$. Also, $a^{\prime} b=b a^{\prime}=a^{\prime} b^{\prime}=b^{\prime} a^{\prime}=0$, so $\left(1+a^{\prime}\right)^{m}$ and $h$ commute and $(1+a)^{m}$ and $1+b^{\prime}$ commute. Thus $g^{m}$ and $h$ commute iff $(1+a)^{m}$ and $1+b$ commute iff $m^{-1} a m b=b m^{-1} a m$. We note that $h_{\top \beta}^{p}\left(h_{\gamma \perp}^{t^{\prime}}-h_{\beta \perp}^{r_{\beta} t^{\prime}}\right)=0=h_{\top \beta}^{p^{\prime}}\left(h_{\gamma \perp}^{t}-h_{\beta \perp}^{r_{\beta} t}\right)$ both if $\beta=\gamma$ (since $t^{\prime}=t=0$ ) and if $\beta \succ \gamma$ (by Lemma 5.1).

We calculate

$$
\begin{gathered}
m^{-1} a m b=\left(1+c^{*}\right) a(1+c) b=\left(h_{\top \beta}^{p}+h_{\top \gamma}^{q}\right)(1+c) b \\
=h_{\top \beta}^{p} h_{\beta \perp}^{s^{\prime}}+h_{\top \gamma}^{q} h_{\gamma \perp}^{t^{\prime}}+h_{\top \beta}^{p} c h_{\gamma \perp}^{t^{\prime}}=h_{c}
\end{gathered}
$$

and

$$
\begin{aligned}
b m^{-1} a m & =b\left(1+c^{*}\right) a(1+c)=b\left(1+c^{*}\right)\left(h_{\beta \perp}^{s}+h_{\gamma \perp}^{t}-h_{\beta \perp}^{r_{\beta} t}\right) \\
& =h_{\top \beta}^{p^{\prime}} h_{\beta \perp}^{s}+h_{\top \gamma}^{q^{\prime}} h_{\gamma \perp}^{t}+h_{\top \beta}^{p^{\prime}} c^{*} h_{\gamma \perp}^{t}=k_{c} .
\end{aligned}
$$

Hence $g^{m}$ and $h$ commute iff $h_{c}=k_{c}$. Letting $c=0$ and applying $h_{c}, k_{c}$ to $v_{\top}$, the result of (a) follows. This also implies (b) in case of $\beta=\gamma$. 
Now let $\beta \succ \gamma$. To show (b), let $x \in R$ and assume $g, h$ commute and $g^{m}, h$ commute, where $m=1+h_{\beta \gamma}^{x}, m^{-1}=1-h_{\beta \gamma}^{x}$. Then $p s^{\prime}+q t^{\prime}=p^{\prime} s+q^{\prime} t$ and $p s^{\prime}+q t^{\prime}+p x t^{\prime}=p^{\prime} s+q^{\prime} t-p^{\prime} x t$, hence $p x t^{\prime}=-p^{\prime} x t$.

Conversely, assume these equalities. To show that $h$ commutes with all conjugates $g^{m}(m \in M)$ of $g$, it suffices to prove that

$$
h_{\top \beta}^{p} c h_{\gamma \perp}^{t^{\prime}}=h_{\top \beta}^{p^{\prime}} c^{*} h_{\gamma \perp}^{t}
$$

for all $m=1+c \in M$. Let $m=1+c \in M$. By Lemma 3.2 we have $v_{\beta} c=-v_{\beta} c^{*} \bmod V_{\gamma}^{-}$ and $v_{\beta} c^{*}=x v_{\gamma}+v^{\prime}$ for some $x \in R, v^{\prime} \in V_{\gamma}^{-}$. So

$$
\begin{aligned}
v_{\top} h_{\top \beta}^{p} c h_{\gamma \perp}^{t^{\prime}} & =p v_{\beta} c h_{\gamma \perp}^{t^{\prime}}=-p v_{\beta} c^{*} h_{\gamma \perp}^{t^{\prime}}=-p x t^{\prime} v_{\perp} \\
& =p^{\prime} x t v_{\perp}=p^{\prime} v_{\beta} c^{*} h_{\gamma \perp}^{t}=v_{\top} h_{\top \beta}^{p^{\prime}} c^{*} h_{\gamma \perp}^{t} .
\end{aligned}
$$

This implies $(+)$.

(c) By Lemma 5.2, we have $p \neq 0 \neq t$, so $p t \neq 0$ since $R$ has no zero-divisors. Hence, by (b), $g$ commutes with all its conjugates iff $p x t=-p x t$ for each $x \in R$ iff $\operatorname{char}(R)=2$.

From now on, let $R$ be commutative. Let $p, q, s, t, p^{\prime}, q^{\prime}, s^{\prime}, t^{\prime} \in R$ with $p \neq 0 \neq t$, and $p s^{\prime}+q t^{\prime}=p^{\prime} s+q^{\prime} t$ and $p t^{\prime}=p^{\prime} t$. The latter equation holds trivially if $t=t^{\prime}=0$, and also in case $p t^{\prime}=-p^{\prime} t$ (cf. Lemma 5.3(b)) and $\operatorname{char}(R)=2$. Now we solve these two linear equations in $Q(R)$. Let $r=\frac{p^{\prime}}{p}=\frac{t^{\prime}}{t} \in Q(R)$, so $p^{\prime}=r p, t^{\prime}=r t$. Hence $p s^{\prime}+q r t=r p s+q^{\prime} t$, so $p\left(s^{\prime}-r s\right)=t\left(q^{\prime}-q r\right)$, thus $\frac{s^{\prime}-r s}{t}=\frac{q^{\prime}-q r}{p}=z \in Q(R)$ showing $s^{\prime}=z t+r s, q^{\prime}=z p+r q$. This motivates part (b) of Definition 5.4.

Definition 5.4. (a) Let $\xi \in \Lambda^{+}$with $\top>\xi>\perp$ and $p, s \in R \backslash\{0\}$.

Then $D_{\xi}^{p s}=\left\langle(1+a)^{U} \mid a=h_{\top \xi}^{r p}+h_{\xi \perp}^{r s}, r \in Q(R), r p, r s \in R\right\rangle$ and $N_{\xi}^{p s}=D_{\xi}^{p s} N_{[\top, \xi),(\xi, \perp]}$. (b) Let $\top>\beta \succ \gamma>\perp$ in $\Lambda^{+}$and $p, q, s, t \in R$. Then put

$$
\begin{aligned}
D_{\beta \gamma}^{p q s t}=\left\langle(1+a)^{U}\right| a=h_{\top \beta}^{r p}+h_{\top \gamma}^{z p+r q}+h_{\beta \perp}^{z t+r s}+h_{\gamma \perp}^{r t}-h_{\beta \perp}^{r_{\beta} r t}, & \\
& \quad r, z \in Q(R), r p, r t, z p+r q, z t+r s \in R\rangle
\end{aligned}
$$

and $N_{\beta \gamma}^{p q s t}=D_{\beta \gamma}^{p q s t} N_{[T, \beta)(\gamma, \perp]}$.

Note that $N_{\lambda}$ is defined for each $\lambda \in \Lambda$, whereas $N_{\xi}^{p s}$ is only defined for $\xi \in \Lambda^{+}$and $N_{\beta \gamma}^{p q s t}$ only if $\beta, \gamma \in \Lambda^{+}$and $\beta \succ \gamma$. 
Observe, for instance $1+h_{\top \beta}^{p}+h_{\top \gamma}^{q}+h_{\beta \perp}^{s}+h_{\gamma \perp}^{t}-h_{\beta \perp}^{r_{\beta} t} \in D_{\beta \gamma}^{p q s t}$ and $1+h_{\top \gamma}^{z p}+h_{\beta \perp}^{z t} \in D_{\beta \gamma}^{p q s t}$ for any $z \in R$.

Proposition 5.5. Let $\top>\xi>\perp$ and $p, s \in R$. Then $N_{\xi}^{p s}$ is an abelian normal subgroup of $U$.

Proof. First we claim that $D_{\xi}^{p s}$ is abelian. Let $g=1+h_{\top \xi}^{r p}+h_{\xi \perp}^{r s}, h=1+h_{\top \xi}^{r^{\prime} p}+h_{\xi \perp}^{r^{\prime} s}$ and $r, r^{\prime} \in Q(R)$ with $r p, r s, r^{\prime} p, r^{\prime} s \in R$. Then $r p r^{\prime} s=r^{\prime} p r s$, so by Lemma 5.3(b), $h$ commutes with all conjugates of $g$. Our claim follows. Since $g$ commutes with all elements of $N_{[\top, \xi),(\xi, \perp]}$, it follows that $N_{\xi}^{p s}$ is abelian.

We note that if $E_{\xi}^{p s}=\left\langle 1+a ; a=h_{\top \xi}^{r p}+h_{\xi \perp}^{r s}, r \in Q(R), r p, r s \in R\right\rangle$, then $N_{\xi}^{p s}=E_{\xi}^{p s} N_{[\top, \xi)(\xi, \perp]}$. Indeed, if $g=1+a$ with $a=h_{\top \xi}^{r p}+h_{\xi \perp}^{r s}$ and $u=1+c \in U$ with $u^{-1}=1+c^{*}$, then $g^{u}=$ $(1+a)^{u}=1+a+b$ with $b=h_{\top \xi}^{r p} c+c^{*} h_{\xi \perp}^{r s}$, so $a b=0$ and $g^{u}=(1+a)(1+b) \in E_{\xi}^{p s} N_{[\top, \xi)(\xi, \perp]}$. Since the elements of $E_{\xi}^{p s}$ commute with those of $N_{[\top, \xi)(\xi, \perp]}$, we obtain $D_{\xi}^{p s} \subseteq E_{\xi}^{p s} N_{[\top, \xi)(\xi, \perp]}$ and our claim. However, this product decomposition is not direct, since $E_{\xi}^{p s} \cap N_{[\top, \xi)(\xi, \perp]}$ contains the element $\left(1+h_{\top \xi}^{p}+h_{\xi \perp}^{s}\right)\left(1+h_{\top \xi}^{-p}+h_{\xi \perp}^{-s}\right)=1+h_{\top \perp}^{-p s} \neq 1$.

Proposition 5.6. $N_{\beta \gamma}^{p q s t}$ is a normal subgroups of $U$. Moreover, $N_{\beta \gamma}^{p q s t}$ is abelian if and only if $\operatorname{char}(R)=2$ or $p=0$ or $t=0$.

Proof. The first statement is clear. Now let $\operatorname{char}(R)=2$ or $p=0$ or $t=0$. We show that $D_{\beta \gamma}^{p q s t}$ is abelian. Let $g=1+a$ with $a=h_{\top \beta}^{r p}+h_{\top \gamma}^{z p+r q}+h_{\beta \perp}^{z t+r s}+h_{\gamma \perp}^{r t}-h_{\beta \perp}^{r_{\beta} r t}$ and $h=1+b$ with $b=h_{\top \beta}^{r^{\prime} p}+h_{\top \gamma}^{z^{\prime} p+r^{\prime} q}+h_{\beta \perp}^{z^{\prime} t+r^{\prime} s}+h_{\gamma \perp}^{r^{\prime} t}-h_{\beta \perp}^{r_{\beta} r^{\prime} t^{\prime}}$ and $r^{\prime}, z^{\prime} \in Q(R)$. Then $r p r^{\prime} t=r^{\prime} p r t$ and $r p\left(z^{\prime} t+r^{\prime} s\right)+(z p+r q) r^{\prime} t=r^{\prime} p(z t+r s)+\left(z^{\prime} p+r^{\prime} q\right) r t$. So by Lemma 5.3(b), $h$ commutes with all conjugates of $g$. Since $g$ also commutes with all elements of $N_{[T, \beta),(\gamma, \perp]}$, it follows that $N_{\beta \gamma}^{p q s t}$ is abelian. Conversely, assume $N_{\beta \gamma}^{p q s t}$ is abelian and $p \neq 0 \neq t$. Then $g=1+h_{\top \beta}^{p}+h_{T_{\gamma}}^{q}+h_{\beta \perp}^{s}+h_{\gamma \perp}^{t}-h_{\beta \perp}^{r_{\beta} t} \in N_{\beta \gamma}^{p q s t}$ with $(\top, \beta),(\gamma, \perp) \in[g]$ by $p \neq 0 \neq t$. Now Lemma 5.3(c) shows that $\operatorname{char}(R)=2$.

Similarly as before, we note that if char $(R)=2$ and

$$
\begin{aligned}
E_{\beta \gamma}^{p q s t}=\left\langle 1+a ; a=h_{\top \beta}^{r p}+h_{\top \gamma}^{z p+r q}+h_{\beta \perp}^{z t+r s}+h_{\gamma \perp}^{r t}-h_{\beta \perp}^{r_{\beta} r t},\right. \\
r, z \in Q(R), r p, r t, z p+r q, z t+r s \in R\rangle,
\end{aligned}
$$


then $N_{\beta \gamma}^{p q s t}=E_{\beta \gamma}^{p q s t} N_{[\top, \beta)(\gamma, \perp]}$, and again this product decomposition is not direct, e.g. if $p \neq 0 \neq t$.

Indeed, consider $g=1+a \in E_{\beta \gamma}^{p q s t}$ with $a=h_{\top \beta}^{r p}+h_{\top \gamma}^{z p+r q}+h_{\beta \perp}^{z t+r s}+h_{\gamma \perp}^{r t}-h_{\beta \perp}^{r_{\beta} r t}$ and $u=1+c \in U$ with $u^{-1}=1+c^{*}$. Then $g^{u}=(1+a)^{u}=1+a+b$ with $b=h_{\top \beta}^{r p} c+$ $h_{\top \gamma}^{z p+r q} c+c^{*}\left(h_{\beta \perp}^{z t+r s}+h_{\gamma \perp}^{r t}-h_{\beta \perp}^{r_{\beta} r t}\right)$. Then $v_{\beta} c=x v_{\gamma}+v^{\prime}$ for some $x \in R$ and $v^{\prime} \in V_{\gamma}^{-}$, so $v_{\top} h_{\top \beta}^{r p} c=r p v_{\beta} c=r p x v_{\gamma}+r p v^{\prime}=v_{\top} h_{\top \gamma}^{r p x}+r p v^{\prime}$. Since $\operatorname{char}(R)=2$, we have $c^{*}=c+c c^{*}$. So $v_{\beta} b=v_{\beta} c h_{\gamma \perp}^{r t}=x v_{\gamma} h_{\gamma \perp}^{r t}=x r t v_{\perp}=v_{\beta} h_{\beta \perp}^{x r t}$. Hence $b=h_{\mathrm{T} \gamma}^{r x p}+h_{\beta \perp}^{r x t}+d$ with $[d] \subseteq[\top, \beta) \times(\gamma, \perp]$, so $g^{u}=\left(1+h_{\top \beta}^{r p}+h_{\top \gamma}^{(z+r x) p+r q}+h_{\beta \perp}^{(z+r x) t+r s}+h_{\gamma \perp}^{r t}-h_{\beta \perp}^{r_{\beta} r t}\right)(1+d) \in E_{\beta \gamma}^{p q s t} N_{[\top, \beta),(\gamma, \perp]}$. Since all elements of $E_{\beta \gamma}^{p q s t}$ commute with those of $N_{[\top, \beta),(\gamma, \perp]}$, we obtain $D_{\beta \gamma}^{p q s t} \subseteq E_{\beta \gamma}^{p q s t} N_{[\top, \beta),(\gamma, \perp]}$ and our claim.

Again this decomposition is not direct. If $p s+q t \neq 0$, let $a$ be as above, with $r=1$ and $z=0$. Then, using Lemma 5.1, $E_{\beta \gamma}^{p q s t} \cap N_{[\top, \beta)(\gamma, \perp]}$ contains $(1+a)^{2}=1+a^{2}=$ $1+h_{\top \beta}^{p} h_{\beta \perp}^{s}+h_{\top \gamma}^{q} h_{\gamma \perp}^{t}=1+h_{\top \perp}^{p s+q t} \neq 1$.

Now let $p s+q t=0$ and consider $g=\prod_{i=1}^{3}\left(1+a_{i}\right)$ with $a_{i}=h_{\top \beta}^{r_{i} p}+h_{\top \gamma}^{z_{i} p+r_{i} q}+h_{\beta \perp}^{z_{i} t+r_{i} s}+h_{\gamma \perp}^{r_{i} t}-$ $h_{\beta \perp}^{r_{\beta} r_{i} t}$ and $r_{1}=r_{2}=z_{2}=z_{3}=1, r_{3}=z_{1}=0$. Then $g \in E_{\beta \gamma}^{p q s t}$ and $\sum r_{i}=\sum z_{i}=0$, so $\sum a_{i}=$ 0 . Hence $g=1+\sum_{i<j} a_{i} a_{j}$ and, again by Lemma 5.1, $a_{i} a_{j}=h_{\top \beta}^{r_{i} p} h_{\beta \perp}^{z_{j} t+r_{j} s}+h_{\top \gamma}^{z_{i} p+r_{i} q} h_{\gamma \perp}^{r_{j} t}=h_{\top \perp}^{x_{i j}}$ with $x_{i j}=\left(r_{i} z_{j}+z_{i} r_{j}\right) p t$ using $p s+q t=0$. But $\sum_{i<j} x_{i j}=3 p t=p t \neq 0$, so $g=1+h_{\top \perp}^{p t} \neq 1$ belongs to $E_{\beta \gamma}^{p q s t} \cap N_{[\top, \beta)(\gamma, \perp]}$.

The following result is the analogue of Theorem 4.8 for the case that $\mathcal{L}^{*}$ is bounded.

Theorem 5.7. Let $\mathcal{L}^{*}$ be a bounded series. Let $N$ be an abelian subgroup of $U$ with normalizer containing $M(\mathcal{L})$. Then $N$ is contained either in some order theoretic normal subgroup $N_{\lambda}$ where $\lambda \in \Lambda$, or in some normal subgroup $N_{\xi}^{p s}$ where $\xi \in \Lambda^{+}, \top>\xi>\perp$ and $p, s \in R \backslash\{0\}$, or, provided that $\operatorname{char}(R)=2$, in some $N_{\beta \gamma}^{p q s t}$ where $\beta, \gamma \in \Lambda^{+}, \top>\beta \succ \gamma>\perp$ and $p, q, s, t \in$ $R, p \neq 0 \neq t$.

Proof. Case 1. First assume that for all $g, h \in N$ and $\alpha \in[g]_{1}, \beta \in[h]_{2}$ we have $\alpha>\beta$.

Following the proof of Theorem 4.8, we obtain a couple $(A, B)$ such that $N \subseteq N_{A, B}$. Then $N_{A, B} \subseteq N_{\xi}$ for some $\xi \in \Lambda$.

Case 2. Assume that there are $g, h \in N$ with $(\top, \xi) \in[g]$ and $(\xi, \perp) \in[h]$, but for each $k \in N$, if $\xi \succ \gamma$ then $(\gamma, \perp) \notin[k]$ and if $\nu \succ \xi$ then $(\top, \nu) \notin[k]$. We will eventually show that $N \subseteq N_{\xi}^{p s}$ for some $p, s \in R \backslash\{0\}$.

Let $k=1+c \in N$. By Lemma 4.7, $[k],[g],[h]$ can only contain the pairs $(\top, \xi),(\xi, \perp)$ and $(\mu, \nu)$ with $\mu>\xi>\nu$. Let $g=1+a$ and $h=1+b$. We may assume that $g$ was chosen 
such that also $(\xi, \perp) \in[g]$. Indeed, suppose $(\xi, \perp) \notin[g]$. If $(\top, \xi) \in[h]$, replace $g$ by $h$. Now let $(\top, \xi) \notin[h]$. Since $(\top, \xi) \in[a]$, there is $v \in V_{\top}^{*}$ with $v a \in V_{\xi}^{*}$. Then $v h=v+v b$ and $v b \in V_{\xi}^{-}, v b a=0$ by the assumption on $[h]$ and $[g]$. Hence $v h a=v a \in V_{\xi}^{*}$ and $(\top, \xi) \in[h a]$. Similarly $(\xi, \perp) \in[b g]$ and we replace $g$ by $h g$.

Hence, by Lemma 5.2 we have

- $a=h_{\top \xi}^{p}+h_{\xi \perp}^{s}+a^{\prime}$,

- $c=h_{\top \xi}^{p^{\prime}}+h_{\xi \perp}^{s^{\prime}}+c^{\prime}$,

- $\left[a^{\prime}\right],\left[c^{\prime}\right] \subseteq[\top, \xi) \times(\xi, \perp]$,

- $p, s, p^{\prime}, s^{\prime} \in R$ and $p \neq 0 \neq s$ by $(\top, \xi),(\xi, \perp) \in[g]$

Now Lemma 5.3(a) implies $p s^{\prime}=p^{\prime} s$, so $\frac{p^{\prime}}{p}=\frac{s^{\prime}}{s}$ in $Q(R)$. Then $k^{\prime}=1+h_{\top \xi}^{p^{\prime}}+h_{\xi \perp}^{s^{\prime}} \in D_{\xi}^{p s}$, showing $k=1+c=k^{\prime}\left(1+c^{\prime}\right) \in N_{\xi}^{p s}$ and so $N \subseteq N_{\xi}^{p s}$.

Case 3. Finally, by Lemma 4.7, it remains to consider the case that there are $g, h \in N$ with $(\top, \beta) \in[g],(\gamma, \perp) \in[h]$ and $\top>\beta \succ \gamma>\perp$. We will show that $N \subseteq N_{\beta \gamma}^{p q s t}$ for some $p, q, s, t \in R$.

Let $k \in N$. Lemma 4.7 implies that $[k],[g],[h]$ each can only contain the pairs $(\top, \beta),(\top, \gamma),(\beta, \perp$ ),$(\gamma, \perp)$ and $(\mu, \nu)$ with $\mu>\beta \succ \gamma>\nu$. We may assume that $g$ was chosen such that also $(\gamma, \perp) \in[g]$. Indeed, suppose that $(\gamma, \perp) \notin[g]$. If $(\top, \beta) \in[h]$, replace $g$ by $h$. If $(\top, \beta) \notin[h]$, clearly, as before, $(\top, \beta),(\gamma, \perp) \in[h g]$ and we replace $g$ by $h g$. Now, by Lemma 5.2 write $g=1+h_{\top \beta}^{p}+h_{\top \gamma}^{q}+h_{\beta \perp}^{s}+h_{\gamma \perp}^{t}-h_{\beta \perp}^{r_{\beta} t}+a^{\prime}$ where $p, q, s, t \in R, p \neq 0 \neq t$ and $\left[a^{\prime}\right] \subseteq[\top, \beta) \times(\gamma, \perp]$. Since $g$ commutes with all its conjugates, by Lemma 5.3(c) we have $\operatorname{char}(R)=2$. We claim that $k \in N_{\beta \gamma}^{p q s t}$. By Lemma 5.2 we can write $k$ in the form $k=1+c+c^{\prime}$ with $c=h_{\top \beta}^{p^{\prime}}+h_{\top \gamma}^{q^{\prime}}+h_{\beta \perp}^{s^{\prime}}+h_{\gamma \perp}^{t^{\prime}}-h_{\beta \perp}^{r_{\beta} t^{\prime}}$ where $p^{\prime}, q^{\prime}, s^{\prime}, t^{\prime} \in R$ and $\left[c^{\prime}\right] \subseteq[\top, \beta) \times(\gamma, \perp]$. Then $k=(1+c)\left(1+c^{\prime}\right)$ and $1+c^{\prime} \in N_{[\top, \beta)(\gamma, \perp]}$, so it remains to show that $1+c \in D_{\beta \gamma}^{p q s t}$. By Lemma 5.3(b) we have $p t^{\prime}=p^{\prime} t$ and $p s^{\prime}+q t^{\prime}=p^{\prime} s+q^{\prime} t$, and the calculations before Definition 5.4 show that $p^{\prime}=r p, t^{\prime}=r t, s^{\prime}=z t+r s, q^{\prime}=z p+r q$ for some $r, z \in Q(R)$. Hence $1+c \in D_{\beta \gamma}^{p q s t}$, showing $N \subseteq N_{\beta \gamma}^{p q s t}$.

Next we show that any two of the normal subgroups $N_{\lambda}, N_{\xi}^{p s}, N_{\beta \gamma}^{p q s t}$ cannot be contained in each other. 
Lemma 5.8. Let $\top>\left\{\xi, \xi^{\prime}\right\}>\perp$ and $p, s, p^{\prime}, s^{\prime} \in R \backslash\{0\}$.

(a) If $N_{\xi}^{p s} \subseteq N_{\xi^{\prime}}^{p^{\prime} s^{\prime}}$, then $\xi=\xi^{\prime}$ and $N_{\xi}^{p s}=N_{\xi}^{p^{\prime} s^{\prime}}$. Furthermore $N_{\xi}^{p s}=N_{\xi}^{p^{\prime} s^{\prime}}$ iff $\frac{p^{\prime}}{p}=\frac{s^{\prime}}{s}$.

(b) If $\lambda \in \Lambda$, then $N_{\xi}^{p s} \nsubseteq N_{\lambda}$ and $N_{\lambda} \nsubseteq N_{\xi}^{p s}$.

Proof. Let $g=1+h_{\top \xi}^{p}+h_{\xi \perp}^{s}$. Then $(\top, \xi),(\xi, \perp) \in[g]$.

(a) Clearly $g \in N_{\xi}^{p s} \subseteq N_{\xi^{\prime}}^{p^{\prime} s^{\prime}}$, so $[g] \subseteq\left[\top, \xi^{\prime}\right] \times\left[\xi^{\prime}, \perp\right]$ and $\xi=\xi^{\prime}$. Also, $g \in N=N_{\xi}^{p^{\prime} s^{\prime}}$, an abelian normal subgroup, and for this situation it was shown in the proof of Theorem 5.7, case 2 , that $N \subseteq N_{\xi}^{p s}$ and $p s^{\prime}=p^{\prime} s$. Conversely, if $p s^{\prime}=p^{\prime} s$ we have $E_{\xi}^{p s}=E_{\xi}^{p^{\prime} s^{\prime}}$ and therefore $N_{\xi}^{p s}=N_{\xi}^{p^{\prime} s^{\prime}}$. (This could also be proved by elementary calculations using the definition of $N_{\xi}^{p s}$.)

(b) Again $g \in N_{\xi}^{p s} \backslash N_{\lambda}$. If $\lambda \geq \xi$, then $h=1+h_{\top \lambda}^{p} \in N_{\lambda}$ and suppose $h \in N_{\xi}^{p s}$. Then $\lambda=\xi$ and $h=1+h_{\top \xi}^{r p}+h_{\xi \perp}^{r s}+a^{\prime}$ for some $r \in Q(R)$ and $\left[a^{\prime}\right] \subseteq[\top, \xi) \times(\xi, \perp]$. Then $a^{\prime}=0$ and $r=1$, but $h_{\xi \perp}^{s} \neq 0$ by $s \neq 0$, a contradiction. If $\xi>\lambda$, then $h=1+h_{\xi \perp}^{s} \in N_{\lambda}$, and if $h \in N_{\xi}^{p s}$, we obtain a contradiction as before.

Lemma 5.9. Assume $\operatorname{char}(R)=2$. Let $\top>\beta \succ \gamma>\perp, \top>\beta^{\prime} \succ \gamma^{\prime}>\perp$ and $p, q, s, t, p^{\prime}, q^{\prime}, s^{\prime}, t^{\prime} \in R$ with $p \neq 0 \neq t, p^{\prime} \neq 0 \neq t^{\prime}$.

(a) If $N_{\beta \gamma}^{p q s t} \subseteq N_{\beta^{\prime} \gamma^{\prime}}^{p^{\prime} q^{\prime} t^{\prime}}$, then $\beta=\beta^{\prime}, \gamma=\gamma^{\prime}$ and $N_{\beta \gamma}^{p q s t}=N_{\beta \gamma}^{p^{\prime} q^{\prime} s^{\prime} t^{\prime}}$. Furthermore, $N_{\beta \gamma}^{p q s t}=$ $N_{\beta \gamma}^{p^{\prime} q^{\prime} s^{\prime} t^{\prime}}$ iff there are some uniquely determined $r, z \in Q(R)$ with $p^{\prime}=r p, t^{\prime}=r t, s^{\prime}=$ $z t+r s, q^{\prime}=z p+r q$.

(b) If $\lambda \in \Lambda$, then $N_{\beta \gamma}^{p q s t} \nsubseteq N_{\lambda}$ and $N_{\lambda} \nsubseteq N_{\beta \gamma}^{p q s t}$.

(c) If $\xi \in \Lambda^{+}$and $p^{\prime} \neq 0 \neq s^{\prime}$, then $N_{\beta \gamma}^{p q s t} \nsubseteq N_{\xi}^{p^{\prime} s^{\prime}}$ and $N_{\xi}^{p^{\prime} s^{\prime}} \nsubseteq N_{\beta \gamma}^{p q s t}$.

Proof. Let $g=1+h_{\top \beta}^{p}+h_{\top \gamma}^{q}+h_{\beta \perp}^{s}+h_{\gamma \perp}^{t}-h_{\beta \perp}^{r_{\beta} t}$.

(a) Clearly $g \in N_{\beta \gamma}^{p q s t} \subseteq N_{\beta^{\prime} \gamma^{\prime}}^{p^{\prime} q^{\prime} t^{\prime}}$, so $[g] \subseteq\left[\top, \gamma^{\prime}\right] \times\left[\beta^{\prime}, \perp\right]$. Since $(T, \beta),(\gamma, \perp) \in[g]$, we obtain $\beta^{\prime} \geq \beta$ and $\gamma \geq \gamma^{\prime}$. Since $\beta \succ \gamma$ and $\beta^{\prime} \succ \gamma^{\prime}$, we get $\beta=\beta^{\prime}$ and $\gamma=\gamma^{\prime}$. Also, $g \in N=N_{\beta \gamma}^{p^{\prime} q^{\prime} s^{\prime} t^{\prime}}$, an abelian normal subgroup, and for this situation it was shown in the proof of Theorem 5.7, case 3, that $N \subseteq N_{\beta \gamma}^{p q s t}$ and $p^{\prime}=r p, t^{\prime}=r t, s^{\prime}=z t+r s, q^{\prime}=z p+r q$ for some $r, z \in Q(R)$. Hence $r=\frac{p^{\prime}}{p}$ and $z=\frac{s^{\prime}-r s}{t}$. Conversely, if $r, z \in Q(R)$ and $p^{\prime}=r p, t^{\prime}=$ $r t, s^{\prime}=z t+r s, q^{\prime}=z p+r q$, we have $E_{\beta \gamma}^{p q s t}=E_{\beta \gamma}^{p^{\prime} q^{\prime} s^{\prime} t^{\prime}}$ and therefore $N_{\beta \gamma}^{p q s t}=N_{\beta \gamma}^{p^{\prime} q^{\prime} s^{\prime} t^{\prime}}$.

(b) Since $(\top, \beta),(\gamma, \perp) \in[g]$, we have $g \in N_{\beta \gamma}^{p q s t} \backslash N_{\lambda}$. First let $\lambda \geq \gamma$. Then $h=1+h_{T_{\gamma}}^{p} \in N_{\lambda}$, and we claim $h \notin N_{\beta \gamma}^{p q s t}$. Indeed, otherwise we would obtain $h=1+h_{\mathrm{T} \beta}^{r p}+h_{\mathrm{T} \gamma}^{z p+r q}+h_{\beta \perp}^{z t+r s}+$ $h_{\gamma \perp}^{r t}-h_{\beta \perp}^{r_{\beta} r t}+a^{\prime}$ for some $r, z \in Q(R)$ and $\left[a^{\prime}\right] \subseteq[\top, \beta) \times(\gamma, \perp]$. Then $a^{\prime}=0$ and $r=0, z=1$, but $h_{\beta \perp}^{t} \neq 0$ by $t \neq 0$, a contradiction. Now let $\gamma>\lambda$. Then $h=1+h_{\beta \perp}^{t} \in N_{\lambda}$, and if 
$h \in N_{\beta \gamma}^{p q s t}$, we obtain a contradiction as before.

(c) Again, $g \in N_{\beta \gamma}^{p q s t} \backslash N_{\xi}^{p^{\prime} s^{\prime}}$. If $\xi \geq \beta$, then $1+h_{\top \gamma}^{p^{\prime}} \in N_{\xi}^{p^{\prime} s^{\prime}} \backslash N_{\beta \gamma}^{p q s t}$ as in (b). If $\gamma \geq \xi$, consider $h=1+h_{\top \xi}^{p^{\prime}}+h_{\xi \perp}^{s^{\prime}} \in N_{\xi}^{p^{\prime} s^{\prime}}$. If $\gamma>\xi$, clearly $h \notin N_{\beta \gamma}^{p q s t}$. Now let $\xi=\gamma$, and suppose $h \in N_{\beta \gamma}^{p q s t}$. Then $h=1+h_{\top \beta}^{r p}+h_{\top \gamma}^{z p+r q}+h_{\beta \perp}^{z t+r s}+h_{\gamma \perp}^{r t}-h_{\beta \perp}^{r_{\beta} r t}+a^{\prime}$ for some $r, z \in Q(R)$ and $\left[a^{\prime}\right] \subseteq[\top, \beta) \times(\gamma, \perp]$. Then $r=0$, but $h_{\xi \perp}^{s^{\prime}}=h_{\gamma \perp}^{r t}$ implies $0 \neq s^{\prime}=r t$ and $r \neq 0$, a contradiction.

Now we can prove the analogue of Corollary 4.9.

Corollary 5.10. Let $\mathcal{L}^{*}$ be bounded. Then the maximal abelian normal subgroups of $U$ are precisely the groups $N_{\lambda}(\lambda \in \Lambda), N_{\xi}^{p s}\left(\xi \in \Lambda^{+}, p, s \in R \backslash\{0\}\right)$ and, provided that char $(R)=2$, $N_{\beta \gamma}^{p q s t}\left(\beta, \gamma \in \Lambda^{+}, \beta \succ \gamma, p, q, s, t \in R, p \neq 0 \neq t\right)$.

Proof. Straightforward by Theorem 5.7 and Lemmas 4.6(b), 5.8, 5.9.

Hence, in comparison with the situation of Corollary 4.9 here we have obtained 'new' maximal abelian normal subgroups. Next we consider intersections of these groups in order to ultimately obtain normal subgroups which determine the elements of $\Lambda^{+}$and thereby the order relation of the chain $\left(\Lambda^{+}, \leq\right)$.

Lemma 5.11. Let $\top>\xi \geq \xi^{\prime}>\perp$ and $p, s, p^{\prime}, s^{\prime} \in R \backslash\{0\}$ with $N_{\xi}^{p s} \neq N_{\xi^{\prime}}^{p^{\prime} s^{\prime}}$. Then: (a) $N_{\xi}^{p s} \cap N_{\xi^{\prime}}^{p^{\prime} s^{\prime}}=N_{[\top, \xi),\left(\xi^{\prime}, \perp\right]}$.

(b) For any $\lambda \in \Lambda$, we have $N_{\xi}^{p s} \cap N_{\lambda}= \begin{cases}N_{[\top, \xi),[\lambda, \perp]} & \text { if } \xi>\lambda, \\ N_{[\top, \lambda),(\xi, \perp]} & \text { if } \lambda \geq \xi .\end{cases}$

Proof. (a) Each element $g \in N_{\xi}^{p s} \cap N_{\xi^{\prime}}^{p^{\prime} s^{\prime}}$ can be written in the form

$$
g=1+h_{\top \xi}^{r p}+h_{\xi \perp}^{r s}+a=1+h_{\top \xi^{\prime}}^{r^{\prime} p^{\prime}}+h_{\xi^{\prime} \perp}^{r^{\prime} s^{\prime}}+a^{\prime}
$$

with $r, r^{\prime} \in Q(R)$ and $[a] \subseteq[\top, \xi) \times(\xi, \perp],\left[a^{\prime}\right] \subseteq\left[\top, \xi^{\prime}\right) \times\left(\xi^{\prime}, \perp\right]$. If $\xi>\xi^{\prime}, r \neq 0$ would imply $(\top, \xi) \in[g]$, and $r^{\prime} \neq 0$ would imply $\left(\xi^{\prime}, \perp\right) \in[g]$, giving in both cases a contradiction. Hence $r=r^{\prime}=0$ and $a=a^{\prime}$, so $g \in N_{[\top, \xi),\left(\xi^{\prime}, \perp\right]}$. Now let $\xi=\xi^{\prime}$. Then $r p=r^{\prime} p^{\prime}$ and $r s=r^{\prime} s^{\prime}$. Now if, say, $r \neq 0$, then $r^{\prime} \neq 0$ and $p=r^{-1} r^{\prime} p^{\prime}, p^{\prime}=\left(r^{\prime}\right)^{-1} r p$ and similarly for $s, s^{\prime}$, showing that $D_{\xi}^{p s}=D_{\xi}^{p^{\prime} s^{\prime}}$, a contradiction. So $r=r^{\prime}=0$ and $g=1+a \in N_{[\top, \xi),(\xi, \perp]}$ as required. The converse inclusion is immediate by Lemma 4.6(b). 
(b) Again, if $g=1+h_{\top \xi}^{r p}+h_{\xi \perp}^{r s}+a \in N_{\xi}^{p s} \cap N_{\lambda}$, we obtain $r=0$. Then $[a] \subseteq([\top, \xi) \times(\xi, \perp$ ]) $\cap([\top, \lambda) \times[\lambda, \perp])$, which implies the inclusions from left to right. The converse is again immediate.

If $\beta, \gamma \in \Lambda^{+}$with $\beta \succ \gamma$ and $p, t \in R \backslash\{0\}$, let

$$
N_{\beta \gamma}^{p t}=\left\langle 1+h_{\top \gamma}^{z p}+h_{\beta \perp}^{z t}: z \in Q(R), z p, z t \in R\right\rangle N_{[\top, \beta)(\gamma, \perp]} .
$$

Lemma 5.12. Let $\operatorname{char}(R)=2$. Let $\top>\beta \succ \gamma>\perp, \top>\beta^{\prime} \succ \gamma^{\prime}>\perp, \beta \geq \beta^{\prime}$, and $p, q, s, t, p^{\prime}, q^{\prime}, s^{\prime}, t^{\prime} \in R$ with $p \neq 0 \neq t, p^{\prime} \neq 0 \neq t^{\prime}$ and $N_{\beta \gamma}^{p q s t} \neq N_{\beta^{\prime} \gamma^{\prime}}^{p^{\prime} s^{\prime} t^{\prime}}$.

(a)

$$
N_{\beta \gamma}^{p q s t} \cap N_{\beta^{\prime} \gamma^{\prime}}^{p^{\prime} q^{\prime} t^{\prime} t^{\prime}}= \begin{cases}N_{[\top, \beta)\left(\gamma^{\prime}, \perp\right]} & \text { if } \beta>\beta^{\prime} \text { or if } \beta=\beta^{\prime} \text { and } p t^{\prime} \neq p^{\prime} t \\ N_{\beta \gamma}^{p t} & \text { if } \beta=\beta^{\prime} \text { and } p t^{\prime}=p^{\prime} t\end{cases}
$$

(b) For any $\lambda \in \Lambda$, we have

$$
N_{\beta \gamma}^{p q s t} \cap N_{\lambda}= \begin{cases}N_{[\top, \beta)[\lambda, \perp]} & \text { if } \gamma>\lambda \\ N_{\beta \gamma}^{p t} & \text { if } \gamma=\lambda \\ N_{[\top, \lambda)(\gamma, \perp]} & \text { if } \lambda \geq \beta\end{cases}
$$

(c) For any $\xi \in \Lambda^{+}$and $p^{\prime}, s^{\prime} \in R \backslash\{0\}$, we have

$$
N_{\beta \gamma}^{p q s t} \cap N_{\xi}^{p^{\prime} s^{\prime}}= \begin{cases}N_{[\top, \beta)(\xi, \perp]} & \text { if } \gamma \geq \xi \\ N_{[\top, \xi)(\gamma, \perp]} & \text { if } \xi \geq \beta\end{cases}
$$

Proof. (a) Clearly, $N_{[\top, \beta)\left(\gamma^{\prime}, \perp\right]}$ is contained in the left hand side of (a). For the second case, let $\beta=\beta^{\prime}$ and $p t^{\prime}=p^{\prime} t$, then $\gamma=\gamma^{\prime}$ and

$$
\begin{aligned}
\left\langle 1+h_{\top \gamma}^{z p}+h_{\beta \perp}^{z t}\right| z \in Q & (R), z p, z t \in R\rangle \\
& =\left\langle 1+h_{\top \gamma^{\prime}}^{z^{\prime} p^{\prime}}+h_{\beta^{\prime} \perp}^{z^{\prime} t^{\prime}} \mid z^{\prime} \in Q(R), z^{\prime} p^{\prime}, z^{\prime} t^{\prime} \in R\right\rangle \subseteq E_{\beta \gamma}^{p q s t} \cap E_{\beta \gamma}^{p^{\prime} q^{\prime} s^{\prime} t^{\prime}} .
\end{aligned}
$$

Now let $g \in N_{\beta \gamma}^{p q s t} \cap N_{\beta^{\prime} \gamma^{\prime}}^{p^{\prime} q^{\prime} t^{\prime}}$. So $g=1+h_{\top \beta}^{r p}+h_{T \gamma}^{z p+r q}+h_{\beta \perp}^{z t+r s}+h_{\gamma \perp}^{r t}-h_{\beta \perp}^{r_{\beta} r t}+a=$ $1+h_{\top \beta^{\prime}}^{r^{\prime} p^{\prime}}+h_{\top \gamma^{\prime}}^{z^{\prime} p^{\prime}+r^{\prime} q^{\prime}}+h_{\beta^{\prime} \perp}^{z^{\prime} t^{\prime}+r^{\prime} s^{\prime}}+h_{\gamma^{\prime} \perp}^{r^{\prime} t^{\prime}}-h_{\beta^{\prime} \perp}^{r_{\beta} r^{\prime} t^{\prime}}+a^{\prime}$ with $r, z, r^{\prime}, z^{\prime} \in Q(R),[a] \subseteq[\top, \beta) \times(\gamma, \perp]$ 
and $\left[a^{\prime}\right] \subseteq\left[\top, \beta^{\prime}\right) \times\left(\gamma^{\prime}, \perp\right]$. First assume $\beta>\beta^{\prime}$. Clearly $r=r^{\prime}=0$ by considering the action of $g$ on $v_{\top}$ and $v_{\gamma^{\prime}}$. Again by the action of $g$ on $v_{\top}$ and $v_{\beta^{\prime}}$, we get $z=z^{\prime}=0$. So $g=1+a=1+a^{\prime} \in N_{[\top, \beta)\left(\gamma^{\prime}, \perp\right]}$. Secondly, let $\beta=\beta^{\prime}$. Then $r \neq 0$ iff $r^{\prime} \neq 0$, and in this case, $(\top, \beta),(\gamma, \perp) \in[g]$, and by the proof of Theorem 5.7, case 3, we get $N_{\beta \gamma}^{p q s t}=N_{\beta \gamma}^{p^{\prime} q^{\prime} s^{\prime} t^{\prime}}$, a contradiction. Hence $r=r^{\prime}=0$. Thus $z p=z^{\prime} p^{\prime}$ and $z t=z^{\prime} t^{\prime}$. So $z^{\prime}\left(p t^{\prime}-p^{\prime} t\right)=0$. Now if $p t^{\prime} \neq p^{\prime} t$, then $z=z^{\prime}=0$ and again $g=1+a=1+a^{\prime} \in N_{[\top, \beta)(\gamma, \perp]}$. If $p t^{\prime}=p^{\prime} t$, then $g=\left(1+h_{\top \gamma}^{z p}+h_{\beta \perp}^{z t}\right)(1+a) \in N_{\beta \gamma}^{p t}$ is as required.

(b) Again, the right-to-left inclusions are clear. Let $g \in N_{\beta \gamma}^{p q s t} \cap N_{\lambda}$. Then $g=1+h_{\top \beta}^{r p}+$ $h_{\top \gamma}^{z p+r q}+h_{\beta \perp}^{z t+r s}+h_{\gamma \perp}^{r t}-h_{\beta \perp}^{r_{\beta} r t}+a$ with $r, z \in Q(R),[a] \subseteq[\top, \beta) \times(\gamma, \perp]$ and $[g] \subseteq[\top, \lambda) \times[\lambda, \perp]$. By the latter property of $[g]$, we get $r=0$. If $z \neq 0$ then $\beta \in[g]_{1}, \gamma \in[g]_{2}$, so $\gamma=\lambda$, and $g \in N_{\beta \gamma}^{p t}$ is as required. If $z=0$, then we have $g=1+a$, and the result follows.

(c) Let $g$ belong to the normal subgroups on the left hand side, so $g=1+h_{\top \beta}^{r p}+h_{\top \gamma}^{z p+r q}+$ $h_{\beta \perp}^{z t+r s}+h_{\gamma \perp}^{r t}-h_{\beta \perp}^{r_{\beta} r t}+a=1+h_{\top \xi}^{r^{\prime} p^{\prime}}+h_{\xi \perp}^{r^{\prime} s^{\prime}}+a^{\prime}$ with $r, z, r^{\prime} \in Q(R),[a] \subseteq[\top, \beta) \times(\gamma, \perp]$ and $\left[a^{\prime}\right] \subseteq[\top, \xi) \times(\xi, \perp]$. Then the second equation for $g$ prohibits $(\top, \beta),(\gamma, \perp) \in[g]$, so $r=0$. If $r^{\prime} \neq 0$, then $(\top, \xi),(\xi, \perp) \in[g]$ by the second equation for $g$. If $(\top, \xi) \in[a]$, then $\gamma>\xi$ contradicting $(\xi, \perp) \in[g]$. Thus $(\top, \xi) \notin[a]$ and $\xi=\gamma$ which implies $(\gamma, \perp) \in[g]$, a contradiction. Hence $r^{\prime}=0$. Then $[g]=\left[a^{\prime}\right]$, so $z \neq 0$ would imply $\beta>\xi>\gamma$, a contradiction. Hence $g=1+a=1+a^{\prime}$ belongs to the normal subgroup on the right hand side. The converse is clear again.

These results will be utilized in the following section.

\section{$6 \quad$ Arbitrary composition series}

Let $R$ be a domain, $\mathcal{L}$ be any direct composition series of the left R-module $\mathrm{V}$, and let $M(\mathcal{L}) \subseteq U \subseteq G(\mathcal{L})$. Here we will prove that $U$ determines the chain $(\mathcal{L}, \subseteq)$ up to isomorphism or anti-isomorphism also it $\mathcal{L}$ is bounded. For this, we further investigate the abelian normal subgroups of $U$.

Definition 6.1. (a) We call a normal subgroup $N$ of $U$ an intersection group, if $N=$ $N_{1} \cap N_{2}$ for two maximal abelian normal subgroups $N_{1}, N_{2}$ of $U$ with $N_{1} \neq N_{2}$.

(b) An intersection group $N$ is maximal, if there is no intersection group $N^{\prime}$ with $N \subsetneq N^{\prime}$.

(c) If $\lambda \in \Lambda^{+}$, let $N_{\lambda}^{-}=N_{(\infty, \lambda)(\lambda,-\infty)}$. 
Note that, if $\lambda \in \Lambda^{+}$, then $N_{\lambda}^{-} \subsetneq N_{\lambda}$.

Proposition 6.2. Let $\mathcal{L}^{*}$ have either no maximal or no minimal element. Then the maximal intersection groups of $U$ are precisely the groups $N_{\lambda}^{-}\left(\lambda \in \Lambda^{+}\right)$. For each $\lambda \in \Lambda^{+}, N_{\lambda}^{-}=$ $N_{\lambda} \cap N_{\rho}$, where $\rho \in \Lambda$ satisfies $\lambda \succ \rho$, and this is the unique way to write $N_{\lambda}^{-}$as intersection of two maximal abelian normal subgroups.

Proof. If $\lambda>\nu>\rho$ in $\Lambda$, then $N_{\lambda} \cap N_{\rho}=N_{(-\infty, \lambda) \cap[\rho, \infty)} \subsetneq N_{\lambda} \cap N_{\nu}$, hence $N_{\lambda} \cap N_{\rho}$ is not maximal. If $\lambda \succ \rho$ in $\Lambda$, then $\lambda \in \Lambda^{+}$and $N_{\lambda} \cap N_{\rho}=N_{\lambda}^{-}$. Clearly $N_{\lambda}^{-}$is a maximal intersection group by Lemma 4.6, and the uniqueness part is clear.

Note that each interval $(\infty, \beta]$ in $\Lambda$ contains $\mu, \nu \in \Lambda$ with $\infty>\mu \succ \nu \geq \beta$; hence $\mu \in \Lambda^{+}$ but either of $\nu \in \Lambda^{+}$and $\nu \notin \Lambda^{+}$could be possible. So, $U$ has 'many' maximal intersection groups.

Corollary 6.3. The maximal intersection groups are precisely the groups of the form $N_{\lambda}^{-}(\lambda \in$ $\left.\Lambda^{+}\right)$or, provided that $\mathcal{L}^{*}$ is bounded and $\operatorname{char}(R)=2, N_{\beta \gamma}^{p t} \quad(\beta \succ \gamma, p, t \in R \backslash\{0\})$.

Proof. If $\mathcal{L}^{*}$ is not bounded, the result follows from Proposition 6.2. Now let $\mathcal{L}^{*}$ be bounded and $\lambda \in \Lambda^{+}$. By Lemma 5.11(b) we have $N_{\lambda}^{-}=N_{\lambda}^{1,1} \cap N_{\lambda}$. Also, if $\operatorname{char}(R)=2$, $\beta \succ \gamma$ and $p, t \in R \backslash\{0\}$, then $N_{\beta \gamma}^{p t}=N_{\beta \gamma}^{p, 0,0, t} \cap N_{\gamma}$ by Lemma 5.12(b). Hence, the description of the maximal abelian normal subgroups of $U$ given by Corollary 5.10 and by Lemma 5.8(b) respectively $5.9(\mathrm{~b}), N_{\lambda}^{-}$and $N_{\beta \gamma}^{p t}$ are intersection groups.

Now let $N$ be an intersection group, so $N=N_{1} \cap N_{2}$ for two different maximal abelian normal subgroups $N_{1}, N_{2}$ of $U$. We distinguish between several cases. First let $N_{1}=N_{\mu}, N_{2}=$ $N_{\nu}$ for some $\mu, \nu \in \Lambda$ with, say, $\mu>\nu$. Choose $\lambda \in \Lambda^{+}$with $\mu \geq \lambda>\nu$. Then $N=N_{\mu} \cap N_{\nu} \subseteq$ $N_{\lambda}^{-}$.

Next assume that $N_{1}=N_{\xi}^{p s}$ and $N_{2}=N_{\xi^{\prime}}^{p^{\prime} s^{\prime}}$ for some $\xi, \xi^{\prime} \in \Lambda^{+}$and $p, s, p^{\prime}, s^{\prime} \in R \backslash\{0\}$. By Lemma 5.11(a), we have $N=N_{\xi}^{p s} \cap N_{\xi^{\prime}}^{p^{\prime} s^{\prime}} \subseteq N_{\xi}^{-}$and $N \subseteq N_{\xi^{\prime}}^{-}$. Now let $N_{2}=N_{\lambda}$ for some $\lambda \in \Lambda$. Then by Lemma 5.11(b) we obtain again $N=N_{\xi}^{p s} \cap N_{\lambda} \subseteq N_{\xi}^{-}$.

Finally, let $\operatorname{char}(R)=2$ and suppose that $N_{1}=N_{\beta \gamma}^{p q s t}$ for some $\beta \succ \gamma$ and $p, q, s, t \in R$ with $p \neq 0 \neq t$. Then by Lemma 5.12 we obtain that $N=N_{\beta \gamma}^{p q s t} \cap N_{2}$ is either contained in $N_{[T, \beta)(\gamma, \perp]} \subseteq N_{\beta}^{-}$or equals $N_{\beta \gamma}^{p t}$. Hence in any case $N$ is either contained in some $N_{\lambda}^{-}$or equals some $N_{\beta \gamma}^{p t}$. 
Now note that clearly $N_{\lambda}^{-} \subseteq N_{u}^{-}$implies $\lambda=\mu$. Also, never $N_{\lambda}^{-} \subseteq N_{\beta \gamma}^{p t}$. Indeed, if $\lambda \geq \beta$, then $1+h_{\top \gamma}^{p} \in N_{\lambda}^{-} \backslash N_{\beta \gamma}^{p t}$, and if $\gamma \geq \lambda$, then $1+h_{\beta \perp}^{t} \in N_{\lambda}^{-} \backslash N_{\beta \gamma}^{p t}$. Also, never $N_{\beta \gamma}^{p t} \subseteq N_{\lambda}^{-}$since $1+h_{T \gamma}^{p}+h_{\beta \perp}^{t} \in N_{\beta \gamma}^{p t} \backslash N_{\lambda}^{-}$. Finally, if $N_{\beta \gamma}^{p t} \subseteq N_{\beta^{\prime} \gamma^{\prime}}^{p^{\prime}}$, we obtain $\beta=\beta^{\prime}, \gamma=\gamma^{\prime}$ and $p t^{\prime}=p^{\prime} t$ as before by Lemma 5.3(b), so $N_{\beta \gamma}^{p t}=N_{\beta \gamma}^{p^{\prime} t^{\prime}}$. Consequently, by the above, it is clear that the groups $N_{\lambda}^{-}\left(\lambda \in \Lambda^{+}\right)$and $N_{\beta \gamma}^{p t}(\beta \succ \gamma, p, t \in R \backslash\{0\})$ constitute all maximal intersection groups.

Lemma 6.4. Let $\mu_{i} \in \Lambda^{+}(i=1,2,3)$ be pairwise different and let $N_{i}=N_{\mu_{i}}^{-}(i=1,2,3)$. Then $N_{1} \cap N_{2} \subseteq N_{3}$ iff $\mu_{3}$ lies between $\mu_{1}$ and $\mu_{2}$

Proof. As for Lemma 4.10.

Now we obtain:

Theorem 6.5. Let $\mathcal{L}_{1}, \mathcal{L}_{2}$ be two composition series such that $\mathcal{L}_{1}^{*}$ is bounded, and let char $(R) \neq$ 2. Let $M\left(\mathcal{L}_{i}\right) \subseteq U_{i} \subseteq \mathcal{G}\left(\mathcal{L}_{i}\right)$ for $i=1,2$, and assume that $U_{1} \cong U_{2}$. Then the chains $\left(\mathcal{L}_{1}, \subseteq\right)$ and $\left(\mathcal{L}_{2}, \subseteq\right)$ are either isomorphic or anti-isomorphic.

Proof. Let $\varphi: U_{1} \rightarrow U_{2}$ be the given isomorphism. Then $\varphi$ maps the maximal intersection groups of $U_{1}$ bijectively onto those of $U_{2}$. By Corollary $6.3, U_{1}$ has maximal intersection groups $N_{\lambda}^{-}\left(\lambda \in \Lambda^{+}\right)$which can be expressed by Lemma 5.11 (b) in (at least) two ways, $N_{\lambda}^{-}=N_{\lambda} \cap N_{\rho}$ where $\lambda \succ \rho$ and $N_{\lambda}^{-}=N_{\lambda}^{1,1} \cap N_{\lambda}$, as intersection of two maximal abelian normal subgroups. By Proposition 6.2, this is impossible for $U_{2}$ if $\mathcal{L}_{2}^{*}$ has either no maximal or no minimal element. Thus $\mathcal{L}_{2}^{*}$ is also bounded. Since $\operatorname{char}(R) \neq 2$, by Corollary $6.3, \varphi$ induces a bijection $\psi: \Lambda_{1}^{+} \rightarrow \Lambda_{2}^{+}$satisfying $\left(N_{\lambda}^{-}\right) \varphi=N_{\lambda \psi}^{-}$for each $\lambda \in \Lambda_{1}^{+}$. By Lemma 6.4, $\psi$ and $\psi^{-1}$ preserve the induced betweenness relations of the chains $\left(\Lambda_{1}^{+}, \leq\right)$and $\left(\Lambda_{2}^{+}, \leq\right)$. Thus $\psi:\left(\Lambda_{1}^{+}, \leq\right) \rightarrow\left(\Lambda_{2}^{+}, \leq\right)$is either an order-isomorphism or anti-isomorphism.

In the first case, $\psi$ clearly extends to an order-isomorphism from $\left(\Lambda_{1}, \leq\right)$ onto $\left(\Lambda_{2}, \leq\right)$.

Now assume that $\psi$ is an anti-isomorphism. Let $\lambda \in \Lambda_{1}^{+}$. We define the component $C_{\lambda}$ of $\lambda$ to be the set of all $\mu \in \Lambda_{1}$ such that the interval between $\lambda$ and $\mu$ is finite. This component is either finite or isomorphic to $\omega$ or $\omega^{*}$ or $\mathbb{Z}$. On each such component $C$ we proceed as follows. If $C \cong \mathbb{Z}$, we let $\pi$ be the mapping $\psi$ on $C$. Now assume $C$ contains a smallest element $\gamma$. Then $\gamma \notin \Lambda_{1}^{+}$. Choose $\beta \in \Lambda_{1}^{+}$with $\beta \succ \gamma$. Note that $C=C_{\beta}$. Then $\beta^{\prime}=\beta \psi \in \Lambda_{2}^{+}$. The goal is to use $\psi$ to construct an anti-isomorphism $\pi: C_{\beta} \rightarrow C_{\beta^{\prime}}$. First, 
there is no $\alpha^{\prime} \in \Lambda_{2}^{+}$with $\alpha^{\prime} \succ \beta^{\prime}$. For, otherwise $\alpha=\alpha^{\prime} \psi^{-1} \in \Lambda_{1}^{+}$would satisfy $\beta \succ \alpha$, hence $\gamma=\alpha \in \Lambda_{1}^{+}$, a contradiction. So, $\beta^{\prime}$ is the largest element of its component in $\Lambda_{2}$. Choose $\gamma^{\prime} \in \Lambda_{2}$ with $\beta^{\prime} \succ \gamma^{\prime}$. Now define $\pi: C_{\beta} \rightarrow C_{\beta^{\prime}}$ by putting $\gamma \pi=\beta^{\prime}$ and $\beta \pi=\gamma^{\prime}$. If $\gamma^{\prime} \notin \Lambda_{2}^{+}$, we have $C_{\beta^{\prime}}=\left\{\beta^{\prime}, \gamma^{\prime}\right\}$ and $C_{\beta}=\{\beta, \gamma\}$. For if there was $\alpha \in \Lambda_{1}^{+}$with $\alpha \succ \beta$, then $\alpha^{\prime}=\alpha \psi \in \Lambda_{2}^{+}$would satisfy $\beta^{\prime} \succ \alpha^{\prime}$, so $\alpha^{\prime}=\beta^{\prime}$, a contradiction. Similarly, if $\gamma^{\prime} \in \Lambda_{2}^{+}$ then $\alpha=\gamma^{\prime} \psi^{-1} \in \Lambda_{1}^{+}$and $\alpha \succ \beta$, and we put $\alpha \pi=\gamma^{\prime}$. Continuing in this way, we obtain an anti-isomorphism $\pi: C_{\beta} \rightarrow C_{\beta^{\prime}}$. If $C$ contains a largest element, we argue dually.

Now the only elements of $\Lambda_{1}$ which do not belong to some component are those $\lambda \in \Lambda_{1}$ for which no $\mu \in \Lambda_{1}$ satisfies $\mu \succ \lambda$ or $\lambda \succ \mu$. But then $A_{\lambda}=[\top, \lambda) \cap \Lambda_{1}^{+}$and $B_{\lambda}=(\lambda, \perp] \cap \Lambda_{1}^{+}$ satisfy $\inf A_{\lambda}=\lambda=\sup B_{\lambda}$, so we can put $\lambda \pi=\sup \left(A_{\lambda} \pi\right)=\inf \left(B_{\lambda} \pi\right)$ using that $\Lambda_{2}$ is Dedekind-complete. In total, $\pi: \Lambda_{1} \rightarrow \Lambda_{2}$ provides the required anti-isomorphism.

Assume $\psi: \Lambda_{1} \longrightarrow \Lambda_{2}$ is an anti-isomorphism satisfying $\Lambda_{1}^{+} \psi=\Lambda_{2}^{+}$. We claim that then for any $\mu, \nu \in \Lambda_{1}$ with $\mu \succ \nu$ there are $\alpha, \beta \in \Lambda_{1}$ with $\alpha \succ \mu \succ \nu \succ \beta$. Indeed, we have $\nu^{\prime}=\nu \psi \succ \mu \psi=\mu^{\prime}$ in $\Lambda_{2}$. Hence $\nu^{\prime} \in \Lambda_{2}^{+}$which implies $\nu \in \Lambda_{1}^{+}$and the existence of $\beta$. Also, $\mu \in \Lambda_{1}^{+}$, so $\mu^{\prime} \in \Lambda_{2}^{+}$and $\mu^{\prime} \succ \alpha^{\prime}$ for some $\alpha^{\prime} \in \Lambda_{2}$. Then $\alpha=\alpha^{\prime} \psi^{-1} \in \Lambda_{1}$ with $\alpha \succ \mu$.

By Theorems 4.11 and 6.5, the case remains where $\mathcal{L}_{1}^{*}$ is bounded and $\operatorname{char}(R)=2$. For this, we will investigate intersections of intersection groups.

Definition 6.6. (a) We call a normal subgroup $N$ of $U$ an intersection group of order 2, if $N=N_{1} \cap N_{2}$ for two maximal intersection groups $N_{1}, N_{2}$ of $U$ with $N_{1} \neq N_{2}$.

(b) An intersection group $N$ of order 2 is maximal if there is no intersection group $N^{\prime}$ of order 2 with $N \subsetneq N^{\prime}$.

Let $\Lambda^{++}=\left\{\beta \in \Lambda^{+} \mid \beta \succ \gamma\right.$ for some $\left.\gamma \in \Lambda^{+}\right\}$. Note that possibly $\Lambda^{++}$is empty. If $\beta \in \Lambda^{++}$and $\beta \succ \gamma$, we let $N_{\beta}^{--}=N_{(\infty, \beta)(\gamma,-\infty)}$.

Proposition 6.7. Let $\mathcal{L}$ be any chain. The maximal intersection groups of order 2 are precisely the groups $N_{\beta}^{--} \quad\left(\beta \in \Lambda^{++}\right)$.

Proof. Let $\beta, \gamma \in \Lambda^{+}$with $\beta \succ \gamma$. Then $N_{\beta}^{--}=N_{\beta}^{-} \cap N_{\gamma}^{-}$is an intersection group of order 2 .

Now let $N$ be a maximal intersection group of order 2, so $N=N_{1} \cap N_{2}$ for two different maximal intersection groups $N_{1}, N_{2}$. First let $N_{1}=N_{\beta}^{-}$and $N_{2}=N_{\gamma}^{-}$with $\beta, \gamma \in \Lambda^{+}$ 
and $\beta>\gamma$. If there is $\delta \in \Lambda$ with $\beta>\delta>\gamma$, there is $\lambda \in \Lambda^{+}$with $\delta \geq \lambda>\gamma$. So $N=N_{\beta}^{-} \cap N_{\gamma}^{-} \subsetneq N_{\beta}^{-} \cap N_{\lambda}^{-}$, contradicting the maximality of $N$. Hence $\beta \succ \gamma$ and $N=N_{\beta}^{--}$ as required.

Now, by Corollary 6.3, we may assume that $\mathcal{L}^{*}$ is bounded, $\operatorname{char}(R)=2$ and $N_{1}=N_{\beta \gamma}^{p t}$ with $\beta, \gamma \in \Lambda^{+}, \beta \succ \gamma$ and $p, t \in R \backslash\{0\}$. Assume that $N_{2}=N_{\lambda}^{-}$for some $\lambda \in \Lambda^{+}$with $\lambda \succ \rho$, say. By Lemma 5.12(b), we obtain

$$
N=N_{\beta \gamma}^{p t} \cap N_{\lambda}^{-}=N_{\beta \gamma}^{p, 0,0, t} \cap N_{\gamma} \cap N_{\lambda} \cap N_{\rho}= \begin{cases}N_{[\top, \beta)(\lambda, \perp]} & \text { if } \gamma \geq \lambda \\ N_{[\top, \lambda)(\gamma, \perp]} & \text { if } \lambda \geq \beta\end{cases}
$$

Hence the maximality of $N$ implies either $\lambda=\gamma$ or $\lambda=\beta$, so $N=N_{\beta}^{--}$.

Finally, let $N_{2}=N_{\beta^{\prime} \gamma^{\prime}}^{p^{\prime} \prime^{\prime}}$ with $\beta^{\prime}, \gamma^{\prime} \in \Lambda^{+}, \beta^{\prime} \succ \gamma^{\prime}$ and $p^{\prime}, t^{\prime} \in R \backslash\{0\}$. We may assume $\beta \geq \beta^{\prime}$. If $\gamma \geq \beta^{\prime}$, Lemma 5.12(b) implies

$$
\begin{aligned}
N & =N_{\beta \gamma}^{p t} \cap N_{\beta^{\prime} \gamma^{\prime}}^{p^{\prime} t^{\prime}}=N_{\beta \gamma}^{p, 0,0, t} \cap N_{\gamma} \cap N_{\beta^{\prime} \gamma^{\prime}}^{p^{\prime}, 0, t^{\prime}} \cap N_{\gamma^{\prime}} \\
& =N_{[\top, \beta)\left(\gamma^{\prime}, \perp\right]} \cap N_{[\top, \gamma)\left(\gamma^{\prime}, \perp\right]}=N_{[\top, \beta)\left(\gamma^{\prime}, \perp\right]} \subsetneq N_{\beta}^{--},
\end{aligned}
$$

a contradiction. Hence $\beta=\beta^{\prime}$. Now, $p t^{\prime}=p^{\prime} t$ would imply

$$
\begin{aligned}
\left\langle 1+h_{\top \gamma}^{z p}+h_{\beta \perp}^{z t} \mid z \in Q(R), z p, z t \in R\right\rangle & \\
& =\left\langle 1+h_{\top \gamma}^{z p^{\prime}}+h_{\beta \perp}^{z t^{\prime}} \mid z \in Q(R), z p^{\prime}, z t^{\prime} \in R\right\rangle,
\end{aligned}
$$

contradicting the assumption $N_{1} \neq N_{2}$. Hence $p t^{\prime} \neq p^{\prime} t$. If $p \neq t$, we have $N_{\beta \gamma}^{p, 0,0, t} \neq N_{\beta \gamma}^{p, 1,1, t}$ by Lemma 5.9 (a) and so $N_{\beta \gamma}^{p t}=N_{\beta \gamma}^{p, 0,0, t} \cap N_{\beta \gamma}^{p, 1,1, t}$ by Lemma 5.12 (a). If $p=t$, we have $N_{\beta \gamma}^{p, 0,0, t} \neq N_{\beta \gamma}^{p, 1,0, t}$ by Lemma 5.9 (a) and so $N_{\beta \gamma}^{p t}=N_{\beta \gamma}^{p, 0,0, t} \cap N_{\beta \gamma}^{p, 1,0, t}$ by Lemma 5.12 (a). The same argument applies to $N_{\beta \gamma}^{p^{\prime} t^{\prime}}$. Putting these intersections together, by Lemma 5.12 (a) we obtain $N=N_{[\top, \beta)(\gamma, \perp]}=N_{\beta}^{--}$, as required.

Hence all maximal intersection groups of order 2 are of the form $N_{\beta}^{--}$with $\beta \in \Lambda^{++}$. By Lemma 4.6, it is clear that these groups $N_{\beta}^{--}$are maximal of order 2.

We have obtained in the course of the proof that each maximal intersection group $N_{\beta}^{--}$ can be obtained only in one of the following ways:

- $N_{\beta}^{--}=N_{\beta}^{-} \cap N_{\gamma}^{-}$,

- $N_{\beta}^{--}=N_{\beta \gamma}^{p t} \cap N_{\beta}^{-}$or $N_{\beta}^{--}=N_{\beta \gamma}^{p t} \cap N_{\gamma}^{-}$, or 
- $N_{\beta}^{--}=N_{\beta \gamma}^{p t} \cap N_{\beta \gamma}^{p^{\prime} t^{\prime}}$ with $p t^{\prime} \neq p^{\prime} t$ and $p, t, p^{\prime}, t^{\prime} \in R \backslash\{0\}$,

in each case with $\beta, \gamma \in \Lambda^{+}$such that $\beta \succ \gamma$. Hence, if $\nu \in \Lambda^{+}$, and $N_{\nu}^{-}$contains a maximal intersection group of order 2, this group can only be $N_{\nu}^{--}$if $\nu \in \Lambda^{++}$, or $N_{\mu}^{--}$where $\mu \succ \nu$. Similarly, $N_{\beta \gamma}^{p t}$ can only contain $N_{\beta}^{--}$.

In other words: Let $\Lambda^{0}=\left\{\beta \in \Lambda^{+} \backslash \Lambda^{++}\right.$: there is no $\lambda \in \Lambda$ with $\left.\lambda \succ \beta\right\}$. Observe that possibly $\Lambda^{0}$ is empty. If $\beta, \gamma \in \Lambda^{+}$with $\beta \succ \gamma$ (then $\gamma \notin \Lambda^{0}$, and possibly $\gamma \in \Lambda^{+} \backslash \Lambda^{++}$, i.e. $\gamma \succ \delta$ with $\delta \in \Lambda \backslash \Lambda^{+}$), then both $N_{\beta}^{-}$and $N_{\gamma}^{-}$contain $N_{\beta}^{--}$. If $\beta \in \Lambda^{0}$, then there is $N_{\beta}^{-}$, but no $N_{\lambda}^{--}$with $N_{\lambda}^{--} \subseteq N_{\beta}^{-}$.

Hence the groups $N_{\beta}^{-}\left(\beta \in \Lambda^{0}\right)$ are precisely the maximal intersection groups which do not contain a maximal intersection group of order 2 .

Recall that possibly $\Lambda^{++}=\emptyset$ or $\Lambda^{0}=\emptyset$. However, $\Lambda^{++} \cup \Lambda^{0}$ is dense in $\Lambda$. Indeed, since $\mathcal{L}$ is a composition series, for any $\alpha, \delta \in \Lambda$ with $\alpha>\delta$, there are $\beta, \gamma \in \Lambda$ with $\alpha \geq \beta \succ \gamma \geq \delta$. So $\beta \in \Lambda^{+}$. If $\beta \notin \Lambda^{0}$, either $\beta \in \Lambda^{++}$or there is $\mu \in \Lambda$ with $\alpha \geq \mu \succ \beta$. Then $\mu \in \Lambda^{++}$. In any case, there is $\mu \in \Lambda^{++} \cup \Lambda^{0}$ with $\alpha \geq \mu>\delta$.

Lemma 6.8. Let $\mu_{i} \in \Lambda^{++} \cup \Lambda^{0}(i=1,2,3)$ be pairwise different. Let $N_{i}=N_{\mu_{i}}^{--}$if $\mu_{i} \in \Lambda^{++}$, and $N_{i}=N_{\mu_{i}}^{-}$if $\mu_{i} \in \Lambda^{0}(i=1,2,3)$. Then $N_{1} \cap N_{2} \subseteq N_{3}$ iff $\mu_{3}$ lies between $\mu_{1}$ and $\mu_{2}$.

Proof. Observe that if $\alpha, \beta, \gamma \in \Lambda^{+}, \mu \in \Lambda^{0}$ with $\alpha>\beta \succ \gamma$ and $N_{(\infty, \alpha)(\gamma,-\infty)} \subseteq N_{\mu}^{-}$, then $\beta \neq \mu \neq \gamma$ and hence $\alpha \geq \mu>\beta$. Now proceed as for Lemma 4.10, with case distinctions for the different possibilities for each $\mu_{i}$.

Now we obtain our final result:

Theorem 6.9. Let $\mathcal{L}_{1}, \mathcal{L}_{2}$ be two composition series such that $\mathcal{L}_{1}^{*}$ is bounded, and let char $(R)=$ 2. Let $M\left(\mathcal{L}_{i}\right) \subseteq U_{i} \subseteq \mathcal{G}\left(\mathcal{L}_{i}\right)$ for $i=1,2$, and assume that $U_{1} \cong U_{2}$. Then the chains $\mathcal{L}_{1}$ and $\mathcal{L}_{2}$ are either isomorphic or anti-isomorphic.

Proof. Let $\varphi: U_{1} \longrightarrow U_{2}$ be an isomorphism. As shown in the proof of Theorem $6.5, \mathcal{L}_{2}^{*}$ is also bounded. For $i=1,2$, the map $\varphi$ is a bijection from the maximal intersection groups of order $i$ of $U_{1}$ onto those of $U_{2}$, preserving inclusion. By Corollary 6.3, Proposition 6.7 and the above remarks, $\varphi$ induces two bijections $\psi_{1}: \Lambda_{1}^{0} \rightarrow \Lambda_{2}^{0}$ and $\psi_{2}: \Lambda_{1}^{++} \rightarrow \Lambda_{2}^{++}$with $N_{\lambda}^{-} \varphi=N_{\lambda \psi_{1}}^{-}$for $\lambda \in \Lambda_{1}^{0}$ and $N_{\lambda}^{--} \varphi=N_{\lambda \psi_{2}}^{--}$for $\lambda \in \Lambda_{1}^{++}$. By Lemma 6.8,

$$
\psi=\psi_{1} \cup \psi_{2}:\left(\Lambda_{1}^{++} \cup \Lambda_{1}^{0}, \leq\right) \rightarrow\left(\Lambda_{2}^{++} \cup \Lambda_{2}^{0}, \leq\right)
$$


is either an isomorphism or anti-isomorphism. Observe that each element of $\Lambda_{1}^{+} \backslash \Lambda_{1}^{++}$is the infimum of a subset of $\Lambda_{1}^{++} \cup \Lambda_{1}^{0}$. The structure of $\left(\Lambda_{1}, \leq\right)$ is hence completely determined by $\left(\Lambda_{1}^{++} \cup \Lambda_{1}^{0}, \leq\right)$.

Hence, if $\psi$ is an isomorphism, it extends (uniquely) to an isomorphism of $\left(\Lambda_{1}, \leq\right)$ to $\left(\Lambda_{2}, \leq\right)$

If $\psi$ is an anti-isomorphism, we can argue similarly as in the proof of Theorem 6.5 and we obtain an anti-isomorphism $\pi$ from $\Lambda_{1}$ onto $\Lambda_{2}$.

We note that if in the above proof $\pi: \Lambda_{1} \longrightarrow \Lambda_{2}$ is an anti-isomorphism, then $\Lambda_{1}^{+} \pi=\Lambda_{2}^{+}$. So the remark after Theorem 6.5 shows that whenever $\mu \succ \nu$ in $\Lambda_{1}$, there are $\alpha, \beta \in \Lambda_{1}$ with $\alpha \succ \mu \succ \nu \succ \beta$.

\section{References}

[1] P. Conrad, The group of order preserving automorphisms of an ordered abelian group, Proc. Amer. Math. Soc. 9 (1958) 382 - 389.

[2] P. Conrad, A correction and improvement of a theorem on ordered groups, Proc. Amer. Math. Soc. 10 (1959) $182-184$.

[3] A. L. S. Corner and R. Göbel, Prescribing endomorphism algebras - A unified treatment, Proc. London Math. Soc. (3) 50 (1985), 447 - 479.

[4] M. Droste and R. Göbel, McLain groups over arbitrary rings and orderings, Math. Proc. Camb. Phil. Soc. (1995) 117439 - 467.

[5] M. Droste and R. Göbel, The automorphism group of Hahn groups, In: "Ordered Algebraic Structures" (W. C. Holland, J. Martinez, eds.), Kluwer Academic Publishers, 1997, pp. $183-215$.

[6] P. Eklof and A. Mekler, Almost free modules, set-theoretic methods (revised edition), North-Holland, Elsevier, Amsterdam, 2002.

[7] L. Fuchs, Abelian Groups, vol. 1 and 2, (Academic Press (1970 and 1973).

[8] L. Fuchs and L. Salce, Modules over non-Noetherian Domains, Math. Surveys, vol. 84 (Amer. Math. Soc., 2001). 
[9] R. Göbel and J. Trlifaj, Approximations and endomorphism algebras Expositions in Math., 41, Walter de Gruyter, Berlin 2006.

[10] R. Göbel and B. Wald, Separable torsion-free modules of small type, Houston Journal of Math. (1990) $16271-287$.

[11] T. Jech, Set Theory, Academic Press (1978).

[12] F. Leinen and O. Puglisi, Unipotent finitary linear groups, J. London Math. Soc. (2) 48 (1993) $59-76$.

[13] U. Meierfrankenfeld, R. E. Phillips and O. Puglisi, Locally solvable finitary linear groups, J. London Math. Soc. (2) 47 (1993) 31 - 40.

[14] R. Nunke, On direct products of infinite cyclic groups, Proc. Amer. Math. Soc. 13, (1962) $66-71$.

[15] O. Puglisi, Maximal unipotent subgroups of finitary linear groups, J. Algebra 181 (1996) $628-658$.

[16] R. E. Phillips, The structure of groups of finitary transformations, J. Algebra 119 (1988) $400-448$.

[17] J. E. Roseblade, The automorphism group of McLain's characteristically simple group, Math. Zeitschr. 82 (1963), $267-282$.

Manfred Droste

Institut für Informatik,

Universität Leipzig,

04009 Leipzig, Germany

e-mail: droste@informatik.uni-leipzig.de

and

Rüdiger Göbel

Fachbereich 6, Mathematik und Informatik

Universität Duisburg Essen,

45117 Essen, Germany

e-mail: ruediger.goebel@uni-due.de 